Claremont Colleges

Scholarship@ Claremont

Pomona Faculty Publications and Research

Pomona Faculty Scholarship

$1-1-2006$

\title{
Conjugation and Clark Operators
}

Stephan Ramon Garcia

Pomona College

\section{Recommended Citation}

Garcia, S.R., Conjugation and Clark operators, Contemp. Math. 393 (2006), 67-112. MR2198373 (2007b:47073). doi: 10.1090/ conm $/ 393$

This Book Chapter is brought to you for free and open access by the Pomona Faculty Scholarship at Scholarship @ Claremont. It has been accepted for inclusion in Pomona Faculty Publications and Research by an authorized administrator of Scholarship @ Claremont. For more information, please contact scholarship@cuc.claremont.edu. 
Contemporary Mathematics

Volume 393, 2006

\title{
Conjugation and Clark operators
}

\author{
Stephan Ramon Garcia
}

\begin{abstract}
Dedicated to Joseph A. Cima, on the occasion of his 70th birthday.
\end{abstract}
ABSTRACT. We discuss the application of antilinear symmetries (conjugation operators) to problems connected to the compressed shift on the spaces $H^{2} \ominus \varphi H^{2}$ where $\varphi$ denotes a nonconstant inner function. For example, we discuss an explicit parameterization of the noncyclic functions for the backward shift operator and show that compressed shifts and their associated Clark operators [10] belong to the class of complex symmetric operators $[\mathbf{2 3}, \mathbf{2 4}]$. Furthermore, we show that the partial isometry in the polar decomposition of an (invertible) compressed shift is a certain Clark unitary operator. Moreover, we are able to compute certain preferred orthonormal bases for the spaces $H^{2} \ominus \varphi H^{2}$ (where $\varphi$ denotes a nonconstant inner function) that respect the underlying symmetry. This is a self-contained exposition of several results of the author and others contained in the papers $[19,21,23,24,25]$.

\section{Introduction}

The operators of our present interest (namely compressed shifts and their associated Clark perturbations) belong to a surprisingly large class of operators, known collectively as complex symmetric operators. Although there are several different characterizations of such operators, the simplest is in terms of matrix representations: A bounded operator on a Hilbert space is complex symmetric if it has a symmetric matrix representation with respect to some orthonormal basis. At the outset, it is not obvious that compressed shifts should have such matrix representations, nor is it obvious how to go about finding such a preferred orthonormal basis. These problems roughly constitute the topic of this note.

2000 Mathematics Subject Classification. 30D55, 47A15.

Key words and phrases. Complex symmetric operators, shift operators, compressed shift, backward shift, inner functions, Clark perturbations, Jordan operators, Volterra operator. 
There are many concrete examples (besides compressed shifts) of complex symmetric operators and the classical roots of the theory are deep. This surprisingly large class includes all normal operators, all Hankel operators, all finite Toeplitz matrices, and the Volterra operator (see below). Section 2 contains some basic background material on complex symmetric operators, beginning with a brief discussion of the classical theory of complex symmetric matrices (Subsection 2.1). In particular, we highlight a few places where such matrices appear in function theory and function-related operator theory.

From an operator theoretic viewpoint, we prefer things to be coordinatefree. Indeed, we are more interested in operators themselves rather than their matrix representations and therefore a more intrinsic definition of the transpose is necessary. By exploiting the symmetry of a finite Toeplitz matrix with respect to the second diagonal (Subsection 2.2), we motivate a coordinate-free approach to the transpose symmetry $T=T^{t}$ enjoyed by a complex symmetric matrix. This entails the consideration of certain antilinear symmetries which are analogous to complex conjugation (Subsection 2.3). To be specific, we say that an antilinear linear operator $C$ on a complex Hilbert space $\mathcal{H}$ is a conjugation operator (or simply a conjugation) if $C^{2}=I$ and $\langle C f, C g\rangle=\langle g, f\rangle$ for all $f, g$ in $\mathcal{H}$. The standard example of a conjugation operator is complex conjugation on a Lebesgue space $L^{2}(X, \mu)$, where no explicit reference is made to an orthonormal basis.

For a fixed conjugation operator $C$, we define the transpose of an operator by the formula $T^{t}=C T^{*} C$ (Subsection 2.4) and we say that $T$ is $C$-symmetric if $T=C T^{*} C$. Furthermore, we say that $T$ is complex symmetric if there exists a conjugation $C$ such that $T$ is $C$-symmetric (Subsection 2.5). A straightforward computation (see Lemma 2.7) shows that an operator $T$ is complex symmetric if and only if it has a symmetric matrix representation with respect to some orthonormal basis.

The model spaces $H^{2} \ominus \varphi H^{2}$ (where $\varphi$ denotes a nonconstant inner function) possess their own natural conjugation operators. In Section 3 , we study function theoretic aspects of the conjugation operator

$$
C f=\overline{f z \varphi}
$$

on $H^{2} \ominus \varphi H^{2}$. We first review several basic definitions (Subsection 3.1) before examining the connection with the theory of pseudocontinuations. It turns out that conjugation on $H^{2} \ominus \varphi H^{2}$ and pseudocontinuations are closely related concepts and that they represent two different ways of looking at the same underlying phenomenon (Subsection 3.2).

Since each space $H^{2} \ominus \varphi H^{2}$ is a reproducing kernel Hilbert space, it is natural to investigate the relationship between its reproducing kernel $K_{\lambda}$ and $C$. This leads naturally to the conjugate kernel $C K_{\lambda}$ which reproduces the values of $[C f](\lambda)$ via the formula $[C f](\lambda)=\left\langle C K_{\lambda}, f\right\rangle$ (Subsection 3.3). 
Specifically, these functions are given by the formulas

$$
K_{\lambda}(z)=\frac{1-\overline{\varphi(\lambda)} \varphi(z)}{1-\bar{\lambda} z}, \quad\left[C K_{\lambda}\right](z)=\frac{\varphi(z)-\varphi(\lambda)}{z-\lambda},
$$

where $\lambda$ and $z$ belong to the open unit disk $\mathbb{D}$. They play a major role in our computations involving the compressed shift and its associated Clark perturbations.

Our first application of this approach is in the explicit function theoretic parameterization of the space $H^{2} \ominus \varphi H^{2}$ (Subsection 3.4), thereby obtaining a characterization of noncyclic functions for the backward shift on $H^{2}$. In light of a famous theorem of Douglas, Shapiro, and Shields ([13, Thm. 2.2.1]), this also yields a description of $H^{2}$ functions which are pseudocontinuable of bounded type.

Unlike many standard Hilbert spaces, the model spaces do not come equipped with "convenient" orthonormal bases (Subsection 3.5). In light of the conjugation operator $C$, we wish to construct orthonormal bases for the spaces $H^{2} \ominus \varphi H^{2}$ which are also fixed by $C$. We refer to such bases as $C$-real orthonormal bases and the remaining sections of this note are concerned with various aspects of this construction.

Section 4 is essentially an extended example in which we explicitly construct an infinite family of $C$-real bases for the space $H^{2} \ominus \varphi H^{2}$ corresponding to the atomic inner function

$$
\varphi(z)=\exp \left(\frac{z+1}{z-1}\right) .
$$

Our main tool is a certain integral transform $\mathcal{W}: L^{2}[0,1] \longrightarrow H^{2} \ominus \varphi H^{2}$ which arose in Sarason's solution [42] to the Gelfand problem [26] (i.e. characterize the invariant subspace lattice for the Volterra integration operator

$$
[V f](x)=\int_{0}^{x} f(t) d t
$$

on $\left.L^{2}[0,1]\right)$. Specifically, $\mathcal{W}$ provides a unitary equivalence between the Cayley transform $(I-V)(I+V)^{-1}$ of the Volterra operator and its functional model, the compressed shift on $H^{2} \ominus \varphi H^{2}$ (Subsection 4.1).

As we mentioned earlier, the Volterra operator is a complex symmetric operator. Indeed, it is $\mathcal{C}$-symmetric with respect to the conjugation operator

$$
[\mathcal{C} f](x)=\overline{f(1-x)}
$$

on $L^{2}[0,1]$. By following this symmetry through to the compressed shift on $H^{2} \ominus \varphi H^{2}$, we deduce that the compressed shift is $C$-symmetric where $C=\mathcal{W} \mathcal{W}^{*}$ turns out to be the conjugation operator $C f=\overline{f z} \varphi$ that we have already mentioned.

By first solving the corresponding problem in $L^{2}[0,1]$, we obtain an explicit description of certain natural $C$-real bases for $H^{2} \ominus \varphi H^{2}$ (Subsection 4.2). By studying this particular example, we are able to conjecture what a 
more general solution might look like (Subsection 4.3).

Section 5 concerns Toeplitz operators on $H^{2}$ and their compressions to the spaces $H^{2} \ominus \varphi H^{2}$. After reviewing some basic definitions (Subsection 5.1) and discussing a simple example (Subsection 5.2), we show that the compression of any Toeplitz operator to a subspace of the form $H^{2} \ominus \varphi H^{2}$ is $C$ symmetric with respect to the natural conjugation operator $C$ on $H^{2} \ominus \varphi H^{2}$ (Subsection 5.3).

In particular, the compressed shift $S f=P_{\varphi}(z f)$ (where $P_{\varphi}$ denotes the orthogonal projection from $H^{2}$ onto $H^{2} \ominus \varphi H^{2}$ ) is a $C$-symmetric operator. This observation allows us to employ several general results on complex symmetric operators to problems concerning the compressed shift and (ultimately) its Clark perturbations.

In Section 6, we discuss a variant of the classical polar decomposition (for complex symmetric operators) which generalizes an elegant result of Godič and Lucenko [29]. Their theorem asserts that every unitary operator $U$ decomposes as the product $U=C J$ of two conjugations $C$ and $J$ (Subsection 6.1). This generalizes the simple geometric fact that any planar rotation can be written as the product of two reflections.

For a $C$-symmetric operator $T$, there is a related decomposition. For instance, if $T$ is $C$-symmetric, then we can write $T=C J|T|$ where $J$ is an auxiliary conjugation operator which commutes with the modulus $|T|=\sqrt{T^{*} T}$ and with its spectral projections (Subsection 6.2). This result (from [24]) is the main tool with which we attack compressed shifts and their Clark perturbations.

In Section 7, we compute the decomposition described above for the compressed shift. More generally, we actually consider the compressions $S_{\lambda} f=P_{\varphi}\left(b_{\lambda} f\right)$ of Toeplitz operators whose symbols are the disk automorphisms

$$
b_{\lambda}(z)=\frac{z-\lambda}{1-\bar{\lambda} z}
$$

vanishing at points $\lambda$ in $\mathbb{D}$. The compressed shift corresponds to the special case $\lambda=0$. After collecting some preliminary notation (Subsection 7.1), we compute the modulus $\left|S_{\lambda}\right|$ (Subsection 7.2), and the auxiliary conjugation $J_{\lambda}$ (Subsection 7.3) in the decomposition

$$
S_{\lambda}=C J_{\lambda}\left|S_{\lambda}\right| .
$$

If $S_{\lambda}$ happens to be invertible, then the partial isometry $U_{\lambda}$ in the polar decomposition of $S_{\lambda}$ is actually unitary and has the Godič-Lucenko decomposition $U_{\lambda}=C J_{\lambda}$ (Subsection 7.4). Moreover, $U_{\lambda}$ turns out to be a Clarktype unitary operator, namely a rank-one perturbation of the compressed shift. 
The appearance of Clark perturbations in the polar decomposition of compressed shifts motivates us to consider these perturbations from the complex symmetric perspective. In Section 8, we develop a slight generalization of Clark's theory to produce $C$-real bases for the spaces $H^{2} \ominus \varphi H^{2}$ (subject to some function theoretic restrictions).

Using the explicit Godič-Lucenko decomposition $U_{\lambda, \alpha}=C J_{\lambda, \alpha}$, we compute the eigenvalues and eigenvectors (if any exist) of $U_{\lambda, \alpha}$ (Subsection 8.1). These computations proceed along entirely different lines than Clark's original computations and provide an alternate approach to the calculation of the eigenstructure of Clark operators. Under certain circumstances, it is possible to obtain a $C$-real orthonormal basis for $H^{2} \ominus \varphi H^{2}$ by this method (Subsection 8.2).

Acknowledgments. The author wishes to thank D. Sarason (co-author of [25] and advisor) for his numerous comments and suggestions regarding this note. The author is also grateful to M. Putinar, coauthor of $[\mathbf{2 2 , 2 3 , 2 4}$ and general mentor. Moreover, we are indebted to D.Z. Arov, J.A. Cima, J.W. Helton, D. Khavinson, S. Richter, H.S. Shapiro, and C. Sundberg for their constructive comments during the writing of [23] and/or [24]. The author would especially like to thank G. Karaali for reading a preliminary version of this note.

\section{Complex Symmetric Operators}

As we have mentioned in the introduction, we will make extensive use of complex symmetric operators in our discussion of problems related to compressed shifts and Clark operators. In this section, we discuss the basic properties of this class of operators and provide a few simple examples. Much of the material in this section comes from [23].

2.1. Complex symmetric matrices. Before proceeding to discuss complex symmetric operators in full generality, it is useful to reflect for a moment on the classical theory of complex symmetric matrices (see the texts $[18,32])$. In this context, we can motivate our results and see the connection between symmetric matrices and Toeplitz operators.

We say that a square matrix $T$ (with complex entries) is complex symmetric if $T$ coincides with its transpose $T^{t}$. Obvious examples of such matrices include real symmetric matrices and complex Hankel matrices.

A notable early contribution to the subject was made by Takagi $[47$, Thm. II], who established a useful decomposition (reproved by Hua [33], Jacobson [36], Schur [43], and Siegel [46], among others) for complex symmetric matrices and used it to provide an elegant proof of a holomorphic interpolation theorem of Carathéodory and Fejér [7].

Specifically, this theorem states that if $a_{0}, a_{1}, \ldots, a_{n-1}$ are complex numbers, then there exists an analytic function $f$ on the open unit disk $\mathbb{D}$ satisfying $\|f\|_{\infty} \leq 1$ and whose first $n$ nonnegative Fourier coefficients are 
precisely $a_{0}, a_{1}, \ldots, a_{n-1}$ if and only if the associated $n \times n$ Hankel matrix

$$
\left(\begin{array}{cccc}
a_{n-1} & a_{n-2} & \cdots & a_{0} \\
a_{n-2} & a_{n-3} & \cdots & 0 \\
\vdots & \vdots & & \vdots \\
a_{0} & 0 & \cdots & 0
\end{array}\right)
$$

has operator norm less than or equal to one. In the course of his proof of the Carathéodory and Fejér theorem, Takagi showed that any complex symmetric matrix can be written in the form $U D U^{t}$ where $D$ is a nonnegative diagonal matrix and $U$ is unitary. This elegant decomposition has many applications in matrix theory and linear algebra, some of which are detailed in [32].

In the theory of univalent functions, the Goluzin inequality (see [15, Cor. 9, p.128]) can be phrased in terms of complex symmetric matrices. Specifically, recall that a holomorphic function $f$ on $\mathbb{D}$ is univalent if it is injective and normalized if it satisfies $f(0)=0$ and $f^{\prime}(0)=1$. A normalized univalent function is called schlicht.

It turns out that $f$ is schlicht if and only if for any $n$ distinct complex numbers $z_{1}, z_{2}, \ldots, z_{n}$, the inequality

$$
\left|\sum_{j, k=1}^{n} w_{j} w_{k} \log \left(\frac{z_{j} z_{k}}{f\left(z_{j}\right) f\left(z_{k}\right)} \cdot \frac{f\left(z_{j}\right)-f\left(z_{k}\right)}{z_{j}-z_{k}}\right)\right| \leq \sum_{j, k=1}^{n} w_{j} \overline{w_{k}} \log \frac{1}{1-z_{j} \overline{z_{k}}}
$$

holds for all choices of $w_{1}, w_{2}, \ldots, w_{n}$. In terms of matrices, this represents the majorization

$$
|\langle A w, w\rangle| \leq\langle B w, w\rangle,
$$

for any $w=\left(w_{1}, w_{2}, \ldots, w_{n}\right)$ in $\mathbb{C}^{n}$, of the complex symmetric matrix

$$
[A]_{j k}=\log \left(\frac{z_{j} z_{k}}{f\left(z_{j}\right) f\left(z_{k}\right)} \cdot \frac{f\left(z_{j}\right)-f\left(z_{k}\right)}{z_{j}-z_{k}}\right)
$$

by the positive matrix

$$
[B]_{j k}=\log \frac{1}{1-z_{j} \overline{z_{k}}} .
$$

More information on such hermitian-symmetric inequalities can be found in $[16,17]$.

In physical applications, complex symmetric matrices also appear in the theory of wave propagation in continuous media [45] and in the study of certain chemical reaction problems [5]. Perhaps the ubiquity of complex symmetric matrices can best be seen from the fact that any square complex matrix is similar to a complex symmetric one. We will sketch a proof of this result in our discussion below.

Since our interest in this note lies closer to operator theory than linear algebra, we will develop a coordinate free approach. If $C$ denotes the operation of complex conjugation of a vector in $\mathbb{C}^{n}$ :

$$
C\left(z_{1}, z_{2}, \ldots, z_{n}\right)=\left(\overline{z_{1}}, \overline{z_{2}}, \ldots, \overline{z_{n}}\right),
$$


then the transpose symmetry $T=T^{t}$ of a complex symmetric matrix $T$ is equivalent to the algebraic condition $T=C T^{*} C$, where $T^{*}$ denotes the adjoint matrix of $T$. We will take this equation as our starting point.

2.2. Toeplitz matrices. The algebraic condition $T=C T^{*} C$ satisfied by a complex symmetric matrix is readily extended to the more general setting of bounded operators on a Hilbert space. One advantage to generalizing the transpose symmetry $T=T^{t}$ in this way is that the adjoint operation $T \mapsto T^{*}$ is intrinsic to a given Hilbert space and does not require a preferred orthonormal basis to begin with.

The simple example of a finite Toeplitz matrix described below illustrates that even in finite dimensions, it may not be immediately evident that an operator has a symmetric matrix representation with respect to some orthonormal basis.

Let $T$ denote a Toeplitz matrix

$$
T=\left(\begin{array}{ccccc}
a_{0} & a_{1} & a_{2} & \ldots & a_{n-1} \\
a_{-1} & a_{0} & a_{1} & \ldots & a_{n-2} \\
a_{-2} & a_{-1} & a_{0} & \ldots & a_{n-3} \\
\vdots & \vdots & \vdots & & \vdots \\
a_{-(n-1)} & a_{-(n-2)} & a_{-(n-3)} & \ldots & a_{0}
\end{array}\right)
$$

and observe the symmetry of $T$ with respect to the second diagonal. Although the Toeplitz matrix $T$ is not in general symmetric, it does satisfy the algebraic condition $T=C T^{*} C$ where $C$ denotes the antilinear operator

$$
C\left(z_{0}, z_{1}, \ldots, z_{n-1}\right)=\left(\overline{z_{n-1}}, \overline{z_{n-2}}, \ldots, \overline{z_{0}}\right)
$$

on $\mathbb{C}^{n}$. Clearly $C$ shares many of the fundamental properties of complex conjugation. Indeed, we easily see that $C$ is involutive, meaning that

$$
C^{2}=I
$$

and that $C$ is isometric in the sense that

$$
\langle C f, C g\rangle=\langle g, f\rangle
$$

for any vectors $f, g$ in $\mathbb{C}^{n}$. Moreover, it is not hard to find orthonormal bases of $\mathbb{C}^{n}$ with respect to which $C$ can be represented simply as complex conjugation.

EXAMPLE 2.1. In the case $n=4$, the vectors

$$
\begin{aligned}
& e_{1}=\frac{1}{\sqrt{2}}(1,0,0,1) \\
& e_{2}=\frac{1}{\sqrt{2}}(i, 0,0,-i) \\
& e_{3}=\frac{1}{\sqrt{2}}(0,1,1,0) \\
& e_{4}=\frac{1}{\sqrt{2}}(0, i,-i, 0)
\end{aligned}
$$


form an orthonormal basis of $\mathbb{C}^{4}$ which is fixed by the antilinear involution $C\left(z_{0}, z_{1}, z_{2}, z_{3}\right)=\left(\overline{z_{3}}, \overline{z_{2}}, \overline{z_{1}}, \overline{z_{0}}\right)$. Thus

$$
C\left(a_{1} e_{1}+a_{2} e_{2}+a_{3} e_{3}+a_{4} e_{4}\right)=\overline{a_{1}} e_{1}+\overline{a_{2}} e_{2}+\overline{a_{3}} e_{3}+\overline{a_{4}} e_{4}
$$

for any complex constants $a_{1}, a_{2}, a_{3}, a_{4}$ and hence $C$ is unitarily equivalent to complex conjugation with respect to the standard basis of $\mathbb{C}^{4}$. The reader will not have difficulty generalizing this procedure for other values of $n$.

With respect to such a basis, one can show that the matrix representation of any Toeplitz matrix $T$ is symmetric (see Lemma 2.7 below). In particular, this implies that any (finite) Toeplitz matrix is unitarily equivalent to a complex symmetric matrix. While this result is classical (see $[18,32]$ for background), it is not necessarily well-known outside of linear algebra and matrix analysis circles.

A simple, yet important, example of a finite Toeplitz matrix is an $n \times n$ Jordan block (with eigenvalue 0):

EXAMPLE 2.2. Let $T$ denote the matrix

$$
T=\left(\begin{array}{cccccc}
0 & 1 & & & & \\
& 0 & 1 & & & \\
& & 0 & & & \\
& & & \ddots & & \\
& & & & 0 & 1 \\
& & & & & 0
\end{array}\right) .
$$

Since $T$ is a finite Toeplitz matrix, it satisfies $T=C T^{*} C$ where $C$ is the antilinear operator defined by $(2.3)$.

In particular, the preceding example indicates that every Jordan block is unitarily equivalent to a symmetric matrix. This runs somewhat counter to our intuition since Jordan blocks were specifically designed to cope with situations in linear algebra where symmetry (in a somewhat vague sense) is lacking. Moreover, by considering direct sums of Jordan blocks, one easily obtains the following theorem:

THEOREM 2.3. Every square matrix is similar to a complex symmetric matrix.

Along these lines, symmetric canonical forms for arbitrary square matrices have been investigated by several authors, the earliest (1930) perhaps being Wellstein $[49]$ (see also $[11,44]$ and the texts $[18,32]$ ). We also remark that, since a real symmetric matrix must be self-adjoint, the preceding theorem generally requires the use of complex similarity transformations and complex symmetric matrices. Moreover, Theorem 2.3 implies that there are no restrictions on the Jordan canonical form of a complex symmetric matrix. 
The connection between complex symmetric matrices and operator related function theory begins to reveal itself when one observes that

valent eader $n$.

$$
\left(\begin{array}{cccccc}
0 & 1 & & & & \\
& 0 & 1 & & & \\
& & 0 & & & \\
& & & \ddots & & \\
& & & & 0 & 1 \\
& & & & & 0
\end{array}\right)\left(\begin{array}{c}
a_{0} \\
a_{1} \\
a_{2} \\
\vdots \\
a_{n-2} \\
a_{n-1}
\end{array}\right)=\left(\begin{array}{c}
a_{1} \\
a_{2} \\
a_{3} \\
\vdots \\
a_{n-1} \\
0
\end{array}\right)
$$

and hence an $n \times n$ Jordan block (with eigenvalue 0) represents a "part" of the backward shift operator. We will discuss the connections between complex symmetric matrices, shift operators, and function theory in a later section. First, we must generalize some of these simple observations to the setting of operators on an arbitrary Hilbert space.

2.3. Conjugation operators. In our consideration of finite Toeplitz matrices, the antilinear operator (2.3) arose. With this $C$, we recognized that the symmetry of a Toeplitz matrix with respect to the second diagonal can be represented by the simple algebraic formula $T=C T^{*} C$. We now wish to consider such antilinear symmetries in a more general setting.

If $\mathcal{H}$ is a Hilbert space, then we say that $C$ is a conjugation operator on $\mathcal{H}$ if the following conditions hold:

(a) $C$ is antilinear:

$$
C\left(a_{1} f_{1}+a_{2} f_{2}\right)=\overline{a_{1}} C f_{1}+\overline{a_{2}} C f_{2}
$$

for all $a_{1}, a_{2} \in \mathbb{C}$ and $f_{1}, f_{2} \in \mathcal{H}$.

(b) $C$ is isometric:

for all $f, g \in \mathcal{H}$.

$$
\langle C f, C g\rangle=\langle g, f\rangle
$$

(c) $C$ is involutive: $C^{2}=I$.

The astute reader will note that these are not the "minimal" axioms which will yield the same effect. For instance, condition $(a)$ follows easily from $(b)$ and $(c)$. Nevertheless, we prefer to be more explicit than necessary for the sake of clarity.

EXAMPLE 2.4. The most obvious (and trivial) example of a conjugation operator is simply complex conjugation on the one-dimensional complex Hilbert space $\mathbb{C}$. Indeed, this is exactly the notion that we are attempting to generalize. Other obvious examples are complex conjugation with respect to the standard basis in $\mathbb{C}^{n}(2.1)$ and the conjugation operator (2.3) that arose in our consideration of finite Toeplitz matrices.

EXAMPLE 2.5. Another simple example is given by pointwise conjugation $[C f](x)=\overline{f(x)}$ on a Lebesgue space $L^{2}(X, \mu)$. If our measure space $(X, \mu)$ has some symmetry, then we might take that into account as well. 
For example, we will see that the conjugation operator $[C f](x)=\overline{f(1-x)}$ on $L^{2}[0,1]$ arises in the consideration of the Volterra integration operator (see Lemma 4.1).

Despite a multitude of different manifestations, conjugation operators are actually quite simple objects. As the following easy lemma shows, any conjugation operator can be represented as complex conjugation with respect to a certain orthonormal basis:

LEMMA 2.6. If $C$ is a conjugation operator on $\mathcal{H}$, then there exists an orthonormal basis $\left(e_{n}\right)_{n=1}^{\operatorname{dim} \mathcal{H}}$ of $\mathcal{H}$ such that $C e_{n}=e_{n}$ for all $n$.

We refer to such a basis as a $C$-real orthonormal basis for $\mathcal{H}$. The importance of such a basis lies in the fact that the action of a possibly abstract conjugation operator $C$ can be represeñted simply as complex conjugation with respect to the basis $\left(e_{n}\right)_{n=1}^{\operatorname{dim} \mathcal{H}}$. In other words, any conjugation operator is unitarily equivalent to complex conjugation on an $l^{2}$ space of the appropriate dimension.

2.4. The transpose of an operator. For a fixed conjugation operator $C$ on a Hilbert space $\mathcal{H}$, we define the transpose $T^{t}$ of a bounded linear operator $T: \mathcal{H} \longrightarrow \mathcal{H}$ to be the linear operator

$$
T^{t}=C T^{*} C \text {. }
$$

It is important to note that the definition is coordinate free since it depends only upon the conjugation operator $C$ and not on a particular choice of basis for $\mathcal{H}$. Indeed, one frequently considers function theoretic conjugation operators which are not a priori defined in terms of complex conjugation with respect to an orthonormal basis.

In terms of matrix representations, the following lemma shows that the definition $T^{t}=C T^{*} C$ for the transpose of an operator is justified:

LEMma 2.7. If $\left(e_{n}\right)_{n=1}^{\operatorname{dim} \mathcal{H}}$ is a $C$-real orthonormal basis for $\mathcal{H}$ and $[T]_{j k}$ denotes the $j k$-th entry of the matrix representation for $T$ with respect to the basis $\left(e_{n}\right)_{n=1}^{\operatorname{dim}_{n} \mathcal{H}}$, then $\left[T^{t}\right]_{j k}=[T]_{k j}$ for all $j, k=1,2, \ldots, \operatorname{dim} \mathcal{H}$.

PROOF. The proof is a straightforward computation based on the definition $T^{t}=C T^{*} C$ and the isometric property (b) of conjugation:

$$
\begin{aligned}
{\left[T^{t}\right]_{j k} } & =\left\langle T^{t} e_{j}, e_{k}\right\rangle \\
& =\left\langle C T^{*} C e_{j}, e_{k}\right\rangle \\
& =\left\langle C e_{k}, T^{*} C e_{j}\right\rangle \\
& =\left\langle e_{k}, T^{*} e_{j}\right\rangle \\
& =\left\langle T e_{k}, e_{j}\right\rangle \\
& =[T]_{k j} .
\end{aligned}
$$

Thus the matrix for $T^{t}$ with respect to the basis $\left(e_{n}\right)_{n=1}^{\infty}$ is simply the matrix transpose of the matrix for $T$ with respect to the same basis. 
2.5. Complex symmetric operators. Suppose now that $\mathcal{H}$ is a Hilbert space equipped with a conjugation operator $C$. We say that a bounded operator $T$ is $C$-symmetric if $T^{t}=T$. In other words, $T$ is a $C$-symmetric operator if and only if $T=C T^{*} C$. More generally, we will say that $T$ is complex symmetric if there exists a conjugation operator $C$ such that $T$ is $C$-symmetric.

There are many examples of complex symmetric operators. In fact, they are more common than one might initially imagine.

EXAMPLE 2.8. All normal operators are complex symmetric. Indeed, one need only produce a conjugation $C$ commuting with the spectral measure of the given normal operator. In finite dimensions, this amounts to nothing more than the observation that a normal operator can be represented by a diagonal (and hence symmetric) matrix with respect to a certain orthonormal basis. In this case, $C$ can simply be taken to be complex conjugation with respect to that basis.

In general, it suffices to show that the operator $M_{z}$ of multiplication by the independent variable on a Lebesgue space $L^{2}(\mu)$ (where $\mu$ is a compactly supported Borel measure on $\mathbb{C}$ ) is complex symmetric. In this case, one can immediately verify the equation $M_{z}=C M_{z}^{*} C$ where $C$ denotes complex conjugation on $L^{2}(\mu)$.

EXAMPLE 2.9. Since a Hankel matrix is symmetric, any (finite or infinite) Hankel matrix defines a complex symmetric operator on its associated $l^{2}$ space.

EXAMPLE 2.10. Another family of examples is furnished by considering integral operators whose kernels possess certain functional symmetries. For example, (bounded) integral operators of the form

$$
[T f](x)=\int_{X} K(x, y) f(y) d \mu(y)
$$

whose kernels $K(x, y)$ are symmetric (in the sense that $K(x, y)=K(y, x)$ for all $x, y$ in $X)$ are $C$-symmetric with respect to complex conjugation $[C f](x)=\overline{f(x)}$ on the corresponding Lebesgue space $L^{2}(X, \mu)$.

EXAMPLE 2.11. One can also combine complex conjugation on a Lebesgue space $L^{2}(X, \mu)$ with a measure-preserving geometric symmetry of the underlying measure space $(X, \mu)$. For example, the Volterra integration operator

$$
[V f](x)=\int_{0}^{x} f(t) d t,
$$

defined on $L^{2}[0,1]$, is $C$-symmetric with respect to the conjugation $[C f](x)=$ $\overline{f(1-x)}$ (see Lemma 4.1). We will discuss this example and its relationship to compressed shifts in greater detail in Section 4.

EXAMPLE 2.12. All compressed Toeplitz operators (with which the remainder of this note is concerned) are complex symmetric operators. The details and definitions are postponed until Section 5. 
Although we will not do so in this note, one can also consider unbounded complex symmetric operators as well. More information and references on the unbounded theory can be found in $[\mathbf{2 2}, \mathbf{2 4}]$.

It is natural to ask, for a fixed $C$, what operations preserve the class of $C$-symmetric operators. Multiplication, for instance, does not. Indeed, it is not hard to find examples of $2 \times 2$ symmetric matrices whose product is not symmetric. The following lemma (whose proof is omitted) provides one important method for constructing new complex symmetric operators from old ones:

LEMMA 2.13. If $T$ is a $C$-symmetric operator, then $p(T)$ is $C$-symmetric for any polynomial $p(z)$.

By applying the lemma to the polynomial $p(z)=z-\lambda$, we see that if $T$ is a $C$-symmetric operator, then $\lambda$ is an eigenvalue of $T$ if and only if $\bar{\lambda}$ is an eigenvalue for $T^{*}$. Moreover, it is not hard to see that $C$ provides an antilinear isometric bijection between $\operatorname{ker}(T-\lambda I)^{n}$ and $\operatorname{ker}\left(T^{*}-\bar{\lambda} I\right)^{n}$ for all $\lambda$ in $\mathbb{C}$ and all $n$. This is an example of the "spectral symmetry principle", satisfied by any complex symmetric operator. Loosely put, if $T$ is a complex symmetric operator, then $T$ and $T^{*}$ are mirror images in every way and their spectral structures correspond under complex conjugation.

In the finite dimensional setting, the preceding comments do not amount to much. In infinite dimensions, however, we can easily exclude a wide variety of operators from the complex symmetric class.

EXAMPLE 2.14. The unilateral shift $M_{z}$ on $H^{2}$ is not complex symmetric. Indeed, $M_{z}$ has no eigenvalues while $M_{z}^{*}$ (the backward shift) has many $[\mathbf{8}, \mathbf{3 0}]$. Here is another straightforward proof. If there were a conjugation operator $C$ on $H^{2}$ such that $M_{z}$ was $C$-symmetric, then the equation $C M_{z}=$ $M_{z}^{*} C$ would imply that

$$
I=M_{z}^{*} M_{z}=\left(M_{z}^{*} C\right)\left(C M_{z}\right)=C M_{z} M_{z}^{*} C
$$

and hence $M_{z} M_{z}^{*}=I$. This is absurd since $M_{z} M_{z}^{*}$ is the orthogonal projection onto the subspace $z H^{2}$.

More generally, one can show that $T^{*} T$ and $T T^{*}$ are unitarily equivalent whenever $T$ is complex symmetric [24].

\section{Conjugation on Model Spaces}

The conjugation operator that we are most concerned with in this note resides on the so-called model spaces, which we briefly describe.

3.1. Model spaces. A famous theorem of Beurling characterizes the invariant subspaces of the unilateral shift operator $M_{z}$ on $H^{2}$. It asserts that the proper, nontrivial invariant subspaces for $M_{z}$ are precisely the subspaces

$$
\varphi H^{2}=\left\{\varphi f: f \in H^{2}\right\}
$$


where $\varphi$ is a nonconstant inner function, a bounded analytic function on the unit disk $\mathbb{D}$ with nontangential limiting values of unit modulus a.e. on $\partial \mathbb{D}$. Since inner functions and Beurling's theorem are well-understood, we refer the reader to the standard texts $[14,35]$.

It follows from Beurling's theorem that the proper, nontrivial invariant subspaces for the backward shift operator $M_{z}^{*}$ are precisely the subspaces $H^{2} \ominus \varphi H^{2}$, where $\varphi$ is a nonconstant inner function. We refer to these subspaces as model spaces in light of an important theorem of Sz.-Nagy and Foaiş which roughly states that any Hilbert space contraction $T$ such that $T^{n}$ tends strongly to 0 is unitarily equivalent to a suitable vector-valued analogue of the compressed shift (also called a model or Jordan operator) $S: H^{2} \ominus \varphi H^{2} \longrightarrow H^{2} \ominus \varphi H^{2}$ defined by

$$
S f=P_{\varphi}(z f)^{*}
$$

where $P_{\varphi}$ denotes the orthogonal projection from $H^{2}$ onto $H^{2} \ominus \varphi H^{2}$. We remark also that the hypothesis that $T^{n}$ tends strongly to zero is not restrictive and is meant to exclude the possibility of a unitary direct summand. For further details, the reader may consult the recent text [38].

The following lemma shows that each model space carries a natural conjugation operator:

LEMMA 3.1. If $\varphi$ is a nonconstant inner function, then

$$
C f=\bar{f} z \varphi
$$

defines a conjugation operator on $\mathrm{H}^{2} \ominus \varphi \mathrm{H}^{2}$ which preserves outer factors.

Proof. Let $f$ be an arbitrary function in $H^{2} \ominus \varphi H^{2}$ and consider the function $\overline{f z} \varphi$ in $L^{2}(\partial \mathbb{D})$. Although it appears at first that this is not the boundary function of an analytic function on $\mathbb{D}$, the short computation

$$
\langle\overline{f z} \varphi, \overline{z h}\rangle=\langle\varphi h, f\rangle=0
$$

shows that $\overline{f z} \varphi$ is orthogonal to every anti-analytic function which vanishes at the origin. This implies that $\overline{f z} \varphi$, despite its appearance, belongs to $H^{2}$. The similar computation

$$
\langle\overline{f z} \varphi, \varphi h\rangle=\langle\overline{z h}, f\rangle=0
$$

shows that $\overline{f z} \varphi$ is also orthogonal to $\varphi H^{2}$. In other words, the antilinear operator $C$ defined by (3.2) maps $H^{2} \ominus \varphi H^{2}$ to itself. Since $|\varphi|=1$ a.e. on $\partial \mathbb{D}$, it is not hard to verify conditions $(b)$ and $(c)$ in the definition of a conjugation operator. To see that $C$ also preserves outer factors, observe that $|C f|=|f|$ a.e. on $\partial \mathbb{D}$.

Although the conjugation (3.2) is defined in terms of boundary functions, in the case where $\varphi$ is a finite Blaschke product, the conjugation can be quite explicitly realized. 
EXAMPLE 3.2. Let $\varphi$ denote a finite Blaschke product

$$
\varphi(z)=\prod_{k=1}^{n} \frac{z-\lambda_{k}}{1-\overline{\lambda_{k} z}}
$$

with $n$ (not necessarily distinct) zeros $\lambda_{k}$. It is not hard to show that each function $f$ in $H^{2} \ominus \varphi H^{2}$ is of the form

$$
f(z)=\frac{a_{0}+a_{1} z+\cdots+a_{n-1} z^{n-1}}{\left(1-\overline{\lambda_{1}} z\right) \cdots\left(1-\overline{\lambda_{n}} z\right)}
$$

and that the conjugate function is given simply by

$$
[C f](z)=\frac{\overline{a_{n-1}}+\overline{a_{n-2}} z+\cdots+\overline{a_{0}} z^{n-1}}{\left(1-\overline{\lambda_{1}} z\right) \cdots\left(1-\overline{\lambda_{n}} z\right)} .
$$

In particular, the conjugation operator (2.3) we considered in our discussion of Toeplitz matrices corresponds (under a suitable interpretation) to the special case $\varphi=z^{n}$. Further details about the conjugation operator on finite dimensional model spaces can be found in $[\mathbf{2 0 , 2 3}]$.

In the case of a general inner function, the computations are not quite as simple. However, we will see in Subsection 3.4 that the conjugation operator (3.2) can be used to obtain an explicit function theoretic representation for the space $H^{2} \ominus \varphi H^{2}$.

3.2. Conjugation and pseudocontinuation. In this subsection, we discuss the relationship between the concept of pseudocontinuation, noncyclicity for the backward shift $M_{z}^{*}$, and our conjugation operator (3.2) on the model space $H^{2} \ominus \varphi H^{2}$.

Due to the fact that each space $H^{2} \ominus \varphi H^{2}$ is invariant under the backward shift $M_{z}^{*}$, one says that a function $f$ belonging to one of the spaces $H^{2} \ominus \varphi H^{2}$ is noncyclic since the closed linear span of its iterates under $M_{z}^{*}$ fails to be all of $H^{2}$. On the other hand, one says that a function $f$ is cyclic if it does not belong to a subspace of the form $H^{2} \ominus \varphi H^{2}$. Equivalently, $f$ is cyclic if and only if the closed linear span of its iterates under $M_{z}^{*}$ is $H^{2}$.

A remarkable characterization of noncyclic functions is provided by a theorem of Douglas, Shapiro, and Shields. Their theorem requires a bit of explanation. Let $\mathbb{D}_{e}$ denote the complement of the closed unit disk in the extended complex plane. If $f$ and $\tilde{f}$ are meromorphic functions on $\mathbb{D}$ and $\mathbb{D}_{e}$, respectively, with nontangential limiting values that agree almost everywhere on $\partial \mathbb{D}$, then $f$ and $\tilde{f}$ are called pseudocontinuations of one another. A meromorphic function on $\mathbb{D}_{e}$ is of bounded type if it is the quotient of bounded analytic functions on $\mathbb{D}_{e}$. The following theorem from $[13$, Thm. $2.2 .1]$ relates pseudocontinuations to the backward shift operator:

Theorem 3.3 (Douglas, Shapiro, Shields). A function $f \in H^{2}$ is noncyclic for the backward shift operator if and only if $f$ has a pseudocontinuation to $\mathbb{D}_{e}$ which is of bounded type. 
Letting PCBT denote the class of $H^{2}$ functions which have pseudocontinuations to $\mathbb{D}_{e}$ which are of bounded type, we see that there exists a nonconstant inner function $\varphi$ such that $f$ belongs to $H^{2} \ominus \varphi H^{2}$ if and only if $f$ belongs to $P C B T$. The reader is invited to consult the recent books $[\mathbf{8}, 40]$ for a more detailed discussion of the preceding theorem and its generalizations to other function spaces. In particular, [40, Sect. 6.2. 6.3] contains numerous instructive examples.

The simplest nontrivial examples of pseudocontinuable functions are inner functions:

EXAMPLE 3.4. If $\varphi$ is an inner function, then

$$
\tilde{\varphi}(z)=1 / \overline{\varphi(1 / \bar{z})}
$$

is a pseudocontinuation of $\varphi$ to $\mathbb{D}_{e}$ which is of bounded type.

EXAMPLE 3.5. Functions with isolated branch points on $\partial \mathbb{D}$, such as $\sqrt{1+z}$, do not possess pseudocontinuations at all and are therefore cyclic vectors for the backward shift (see [40, Ex. 6.2.3] for a thorough explanation).

To see how Theorem 3.3 fits in the context of conjugation operators, note that a function $f$ belongs to $H^{2} \ominus \varphi H^{2}$ if and only if there exists a function $g$ in $H^{2}$ such that $f=\overline{g z} \varphi$ holds almost everywhere on $\partial \mathbb{D}$. Indeed, the proof of this fact is essentially contained in our proof of Lemma 3.1. If $f$ is orthogonal to $\varphi H^{2}$, then

$$
0=\langle f, \varphi h\rangle=\langle\bar{\varphi} f, h\rangle
$$

for all $h$ in $H^{2}$. This implies that $\bar{\varphi} f$ is orthogonal to $H^{2}$ and hence $\bar{\varphi} f=\overline{g z}$ for some $g$ in $H^{2}$.

Letting $g^{*}$ denote the function $g^{*}(z)=\overline{g(\bar{z})}$ obtained by conjugating the Taylor coefficients of $g$, we can interpret the equation $f=\bar{g} \bar{z} \varphi$ as saying that the functions $f(z) / \varphi(z)$ on $\mathbb{D}$ and $\frac{1}{z} g^{*}\left(\frac{1}{z}\right)$ on $\mathbb{D}_{e}$ have matching nontangential limits almost everywhere on $\partial \mathbb{D}$.

The approach that we take here is to think of the complementary function $g$ as $C f$, a function belonging to $H^{2} \ominus \varphi H^{2}$ and hence with domain $\mathbb{D}$, as opposed to a function on $\mathbb{D}_{e}$. Indeed, the definition (3.2) of $C$ indicates that

$$
f=\overline{(C f) z \varphi} .
$$

In some sense, we see that Lemma 3.1 (on the existence of $C$ ) is a reinterpretation of the Douglas, Shapiro, and Shields result (Theorem 3.3). We will say considerably more about noncyclic functions in Subsection 3.4.

3.3. Conjugation and the reproducing kernel. In this subsection, we investigate the relationship between the conjugation operator (3.2) on a model space $H^{2} \ominus \varphi H^{2}$ and the reproducing kernel Hilbert space structure of $H^{2} \ominus \varphi H^{2}$. 
Recall that the reproducing kernel for $H^{2}$ is the Szegö kernel

$$
e_{\lambda}(z)=\frac{1}{1-\bar{\lambda} z},
$$

where $\lambda, z$ belong to $\mathbb{D}$. These functions have the property that

$$
f(\lambda)=\left\langle f, e_{\lambda}\right\rangle
$$

for every $f$ in $H^{2}$ and all $\lambda$ in $\mathbb{D}$. In fact, the preceding equation follows directly from the Cauchy integral formula or by considering the Taylor expansions of $f$ and $e_{\lambda}$. It is not hard to derive from (3.4) and the definition of $H^{2} \ominus \varphi H^{2}$ that the equation

$$
f(\lambda)=\left\langle f, K_{\lambda}\right\rangle
$$

holds for every $f$ in $H^{2} \ominus \varphi H^{2}$. Here $K_{\lambda}$ denotes the reproducing kernel

$$
K_{\lambda}(z)=\frac{1-\overline{\varphi(\lambda)} \varphi(z)}{1-\bar{\lambda} z}
$$

for $H^{2} \ominus \varphi H^{2}$.

Since the model space $H^{2} \ominus \varphi H^{2}$ is a reproducing kernel Hilbert space, it is natural to investigate the relationship between the reproducing kernel (3.6) and the conjugation operator (3.2). The reproducing property (3.5) of $K_{\lambda}$ and the isometric property of $C$ imply that

$$
[C f](\lambda)=\left\langle C f, K_{\lambda}\right\rangle=\left\langle C K_{\lambda}, f\right\rangle
$$

for all $f$ in $H^{2} \ominus \varphi H^{2}$ and $\lambda$ in $\mathbb{D}$. In other words, the conjugate kernel function $C K_{\lambda}$ reproduces the values of $C f$ via the formula $[C f](\lambda)=\left\langle C K_{\lambda}, f\right\rangle$. Indeed, the existence of such a conjugate kernel could have been deduced from the Riesz representation theorem since the map $f \mapsto \overline{[C f](\lambda)}$ is a bounded linear functional for every $|\lambda|<1$.

Using the definition (3.2) of $C$, we can give an explicit formula for the conjugate kernel function $C K_{\lambda}$. We need only keep in mind that our computations take place on $\partial \mathbb{D}$, where $\varphi$ is unimodular almost everywhere:

$$
\begin{aligned}
{\left[C K_{\lambda}\right](z) } & =\overline{\left(\frac{1-\overline{\varphi(\lambda)} \varphi(z)}{1-\bar{\lambda} z}\right) \bar{z} \varphi(z)} \\
& =\frac{1-\varphi(\lambda) \overline{\varphi(z)}}{1-\lambda \bar{z}} \cdot \frac{\varphi(z)}{z} \\
& =\frac{\varphi(z)-\varphi(\lambda)}{z-\lambda} .
\end{aligned}
$$

Thus the conjugation kernel function $C K_{\lambda}$ for $H^{2} \ominus \varphi H^{2}$ is none other than the difference quotient (3.7).

The last statement of Lemma 3.1 asserts that $C$ preserves outer factors. In particular, this applies to the functions $K_{w}$ and $C K_{w}$. This is an important fact which we verify directly. For each $\lambda$, the reproducing kernel $K_{\lambda}$ is an outer function, being the quotient of the two outer functions $1-\overline{\varphi(\lambda)} \varphi(z)$ 
and $1-\bar{\lambda} z$. Since $C$ preserves outer factors, it follows that the corresponding conjugate function $C K_{\lambda}$ is the product of $K_{\lambda}$ with an inner function. This is indeed the case:

$$
\begin{aligned}
{\left[C K_{\lambda}\right](z) } & =\frac{\varphi(z)-\varphi(\lambda)}{z-\lambda} \\
& =\frac{\varphi(z)-\varphi(\lambda)}{1-\overline{\varphi(\lambda)} \varphi(z)} \cdot \frac{1-\bar{\lambda} z}{z-\lambda} \cdot \frac{1-\overline{\varphi(\lambda)} \varphi(z)}{1-\bar{\lambda} z} \\
& =\frac{b_{\varphi(\lambda)}(\varphi(z))}{b_{\lambda}(z)} K_{\lambda}(z) .
\end{aligned}
$$

Here $b_{a}$ denotes the disk automorphism

$$
b_{a}(z)=\frac{z-a}{1-\bar{a} z}+
$$

where $a$ belongs to $\mathbb{D}$. In particular, we observe that the inner factor of $C K_{\lambda}$ is the inner function $b_{\varphi(\lambda)}(\varphi(z)) / b_{\lambda}(z)$.

A remarkable thing happens as $|\lambda| \longrightarrow 1$. Let $\zeta$ be a point on $\partial \mathbb{D}$ such that $\varphi$ has a nontangential limiting value at $\zeta$ of unit modulus and suppose that the limit function $K_{\zeta}$ formally obtained by substituting $\zeta$ for $\lambda$ in (3.6) belongs to $H^{2}$ (and hence $H^{2} \ominus \varphi H^{2}$ ). Since $\zeta$ and $\varphi(\zeta)$ are unimodular, we see that

$$
\begin{aligned}
{\left[C K_{\zeta}\right](z) } & =\frac{\varphi(z)-\varphi(\zeta)}{z-\zeta} \\
& =\frac{\varphi(\zeta)}{\zeta} \cdot \frac{1-\overline{\varphi(\zeta)} \varphi(z)}{1-\bar{\zeta} z} \\
& =\bar{\zeta} \varphi(\zeta) K_{\zeta}(z) .
\end{aligned}
$$

Thus, the functions $K_{\zeta}$ and $C K_{\zeta}$ corresponding to a point $\zeta$ on $\partial \mathbb{D}$ differ only by the unimodular constant $\bar{\zeta} \varphi(\zeta)$. Selecting any branch of the square root function, it follows from the antilinearity of $C$ that the functions

$$
(\bar{\zeta} \varphi(\zeta))^{\frac{1}{2}} K_{\zeta}
$$

are fixed by $C$ (whenever they belong to $H^{2}$ ). This is an idea that we will return to several times in this note.

3.4. Description of noncyclic functions. Using the conjugation operator (3.2), we can obtain an explicit function theoretic characterization of the functions in $H^{2} \ominus \varphi H^{2}$. The construction that we pursue in this subsection can be found in [19].

Since $C^{2}=I$, we can split a given function $f$ in $H^{2} \ominus \varphi H^{2}$ into $C$-real and $C$-imaginary parts. Each $f$ in $H^{2} \ominus \varphi H^{2}$ decomposes as

$$
f=\frac{1}{2}(f+C f)+i \frac{1}{2 i}(f-C f)
$$

where the terms $\frac{1}{2}(f+C f)$ and $\frac{1}{2 i}(f-C f)$ are both fixed by $C$. This motivates us to find the fixed points for $C$. 
Suppose that $f$ is fixed by $C$. In other words, $f$ satisfies

$$
f=\overline{f z} \varphi
$$

almost everywhere on $\partial \mathbb{D}$. Now select a point $\zeta$ on $\partial \mathbb{D}$ where $\varphi$ has a nontangential limiting value of unit modulus. Since $\varphi$ is an inner function, almost every $\zeta$ will have this property. We may, without loss of generality, assume that $\varphi(\zeta)=\zeta$ since this may be obtained by multiplying $\varphi$ by a suitable unimodular constant.

The boundary kernel function

$$
K_{\zeta}(z)=\frac{1-\overline{\varphi(\zeta)} \varphi(z)}{1-\bar{\zeta} z}
$$

obtained from (3.6) by letting $\lambda \rightarrow \zeta$ nontangentially yields a function in the Smirnov class $N^{+}$(see $[\mathbf{8}, \mathbf{1 4}]$ for more information). Although $K_{\zeta}$ might not belong to $H^{2}$, it is the quotient of two bounded analytic functions and hence belongs to $N^{+}$.

The appearance of the term $\bar{z} \varphi$ in (3.8) is fortuitous as the following calculation will justify. Using the fact that $\varphi(\zeta)=\zeta$ we see that

$$
\begin{aligned}
& K_{\zeta}(z) \sqrt{K_{\zeta}(z)}=\frac{1-\overline{\varphi(\zeta)} \varphi(z)}{1-\bar{\zeta} z} \cdot \frac{1-\zeta \bar{z}}{1-\varphi(\zeta) \overline{\varphi(z)}} \\
& =\frac{1-\overline{\varphi(\zeta)} \varphi(z)}{1-\varphi(\zeta) \overline{\varphi(z)}} \cdot \frac{1-\zeta \bar{z}}{1-\bar{\zeta} z} \\
& =\overline{\varphi(\zeta)} \varphi(z)\left(\frac{\varphi(\zeta) \overline{\varphi(z)}-1}{1-\varphi(\zeta) \overline{\varphi(z)}}\right) \cdot \zeta \bar{z}\left(\frac{\bar{\zeta} z-1}{1-\bar{\zeta} z}\right) \\
& =\zeta \overline{\varphi(\zeta)} \bar{z} \varphi(z) \\
& =\bar{z} \varphi(z)
\end{aligned}
$$

and thus (3.8) can be written in the symmetric form

$$
f / K_{\zeta}=\overline{f / K_{\zeta}} .
$$

This shows that the function $f / K_{\zeta}$ belongs to $N^{+}$and is real almost everywhere on $\partial \mathbb{D}$. Thus each $f$ in $H^{2} \ominus \varphi H^{2}$ can be written in the form

$$
f(z)=[a(z)+i b(z)] K_{\zeta}(z)
$$

where $a$ and $b$ are functions in $N^{+}$which are real a.e. on $\partial \mathbb{D}$.

A function $f$ belonging to the Smirnov class $N^{+}$is called a real Smirnov function if its boundary function is real valued a.e. on $\partial \mathbb{D}$. The set $R^{+}$ of all real Smirnov functions is a real subalgebra of $N^{+}$that was explicitly described by Helson [31]. He showed that if $\psi_{1}$ and $\psi_{2}$ are relatively prime inner functions such that $\psi_{1}-\psi_{2}$ is outer, then the function

$$
f(z)=i \frac{\psi_{1}+\psi_{2}}{\psi_{1}-\psi_{2}}
$$


is a real Smirnov function and every real Smirnov function arises this way. Although elegant, the representation (3.9) has its limitations. For example, the inner functions $\psi_{1}$ and $\psi_{2}$ are often difficult to identify and there are no general criteria describing when the difference of inner functions is outer. A simple proof of Helson's formula (3.9) can be based on linear fractional transformations and the factorization theory for $N^{+}$[25, Sec. 3].

In [25], Sarason and the author proved that any outer function $F$ belonging to $R^{+}$can be represented as a locally uniformly convergent product

$$
F(z)=|F(0)| \prod_{n=1}^{\infty} \frac{T\left(\varphi_{n}^{+}\right)}{T\left(\varphi_{n}^{-}\right)},
$$

where $T$ denotes the linear fractional transformation

$$
T(z)=i \frac{1-i z}{1+i z},
$$

and the inner functions $\varphi_{n}^{+}$and $\varphi_{n}^{-}$are naturally associated with the boundary values of $\arg F$ on $\partial \mathbb{D}$. The proof of this fact is somewhat technical and relies heavily on the Cauchy $A$-integral (see $[4]$ and the recent book $[9]$ ), due to the fact that $\arg F$, unlike $\log |F|$, might not be integrable on $\partial \mathbb{D}$.

By using the Koebe function $k(z)=z /(1-z)^{2}$ (see [15]), we can reduce the description of functions in $R^{+}$to the preceding case of so-called real outer functions. If $\psi$ is a nonconstant inner function, then $k(\psi)$ belongs to $R^{+}$. Since the inner factor of $k(\psi)$ is precisely $\psi$, the construction of the general function in $R^{+}$can be reduced to the product (3.4). A more detailed description of this reduction can be found in [21].

In short, real Smirnov functions can be described explicitly and reasonably constructively in terms of inner functions. In light of Example 3.4, these computations explain the theorem of Douglas, Shapiro, and Shields (Theorem 3.3). Specifically, pseudocontinuations of bounded type arise from inner functions.

Similar considerations lead to the following parameterization of the invariant subspaces for the backward shift on $H^{p}$ (for $1<p<\infty$ ):

$$
H^{p} \cap \overline{\varphi z H^{p}}=\left\{[a(z)+i b(z)] K_{\zeta}(z): a, b \in R^{+}\right\} \cap H^{p} .
$$

Even further, one can use the same method to parametrize the kernels of Toeplitz operators on $H^{p}$ for $1<p<\infty[\mathbf{1 9}]$.

3.5. C-real bases for model spaces. One of our main concerns in this note is to produce $C$-real (fixed by $C$ ) orthonormal bases for the model spaces $H^{2} \ominus \varphi H^{2}$. Our interest in this problem stems not only from the desire to understand the function theoretic aspects of the conjugation operator (3.2), but also to understand its operator theoretic implications.

Unfortunately, an explicit description of $C$-real bases for model spaces is not always readily apparent. Indeed, finding explicit orthonormal bases for model spaces is nontrivial. The Malmquist-Walsh lemma [48] (see also [37, Lec. V.]) for instance, applies only in the case where $\varphi$ is a Blaschke product. 
Moreover, as the reader may check, this procedure does not produce a $C$-real basis for $H^{2} \ominus \varphi H^{2}$.

Instead, we parallel and generalize the method of Clark [10] which, it turns out, often produces $C$-real bases for model spaces. Those familiar with this method will recognize that there is no mention of the conjugation operator (3.2) in [10]. Indeed, that article was concerned with producing only orthonormal bases for the model spaces. A more detailed study of complex symmetric operators and their properties will ultimately explain exactly why Clark's method often succeeds in producing $C$-real bases.

\section{Conjugation and the Volterra Operator}

In this section, we consider the specific problem of constructing $C$-real bases for the model space $H^{2} \ominus \varphi H^{2}$ where $\varphi$ denotes the singular inner function

$$
\varphi(z)=\exp \left(\frac{z+1}{z-1}\right)
$$

arising from a unit point mass at $z=1$. In other words, we wish to find an orthonormal basis for $H^{2} \ominus \varphi H^{2}$ that is fixed by the conjugation operator $C f=\bar{f} z \varphi$. Finding such a basis is natural from the viewpoint of complex symmetric operators, for the matrix representation of the compression of a Toeplitz operator to $H^{2} \ominus \varphi H^{2}$ will be symmetric with respect to such a basis.

Although we will shortly develop a much more general approach to this problem, a careful analysis of this special case will be instructive. Indeed, this simple case will illustrate some of our main points and highlight the relationship between our approach and several well-known and classical results.

We now consider the relationship between our basis problem and Sarason's solution to the Gelfand problem.

4.1. The Gelfand problem. In 1938, I.M. Gelfand raised the question of characterizing the invariant subspace lattice of the Volterra integration operator

$$
[V f](x)=\int_{0}^{x} f(t) d t
$$

on $L^{2}[0,1][26]$. It is clear that for any $a$ in $[0,1]$, the subspace $\chi_{[0, a]} L^{2}[0,1]$ is an invariant subspace for $V$. Here $\chi_{[0, a]}$ denotes the characteristic function of the closed interval $[0, a]$.

It turns out that these are the only invariant subspaces for the Volterra operator and hence Lat $V$ is linearly ordered, or unicellular. This result was proved first in 1949 by Agmon [1] and later by Sakhnovich [41], Brodskii [6], Donoghue [12], Kalisch [34], and Sarason [42]. We are interested here not in the Gelfand problem itself, but rather in a clever technique used by Sarason, which was later generalized by Ahern and Clark [3] (see also [37, Lec. 5]). 
What Sarason noted was that the lattice of invariant subspaces for the Volterra operator corresponds, under a certain integral transform, to the lattice of invariant subspaces for the compressed shift on the model space $H^{2} \ominus \varphi H^{2}$ corresponding to the atomic inner function

$$
\varphi(z)=\exp \left(\frac{z+1}{z-1}\right) .
$$

Once this is proved, the unicellularity of Lat $V$ follows immediately from Beurling's theorem. Indeed, the inner functions dividing $\varphi$ are precisely the functions $\varphi^{t}$ for $0 \leq t \leq 1$ where $\varphi^{t}$ is unambiguously defined by the formula

$$
[\varphi(z)]^{t}=\exp \left(t \frac{z+1}{z-1}\right) .
$$

The integral transform *

$$
[\mathcal{W} g](z)=\frac{\sqrt{2} i}{z-1} \int_{0}^{1} g(t)[\varphi(z)]^{t} d t
$$

is a unitary map from $L^{2}[0,1]$ to $H^{2} \ominus \varphi H^{2}$. Indeed, this is simply a unimodular constant multiple of the transformation appearing in the introduction to Clark's original paper [10] on compressed shifts. Although at first the formula (4.1) might appear a little mysterious, it is easy to motivate once one observes that the integrand $g(t) \varphi^{t}(z)$ (as a function of $z$ ) belongs to $H^{2} \ominus \varphi H^{2}$ for all $t$ in $[0,1]$. In fact, the operator $\mathcal{W}$ yields the unitary equivalence of the characteristic function of the Cayley transform

$$
(I-V)(I+V)^{-1}
$$

of the Volterra operator $V$ with its functional model, the compressed shift $S$ on $H^{2} \ominus \varphi H^{2}$ (for full details, see [38] and [37, Lec. 5]). In other words, the operators $S$ and $V$ are related by the formula

$$
S=\mathcal{W}\left[(I-V)(I+V)^{-1}\right] \mathcal{W}^{*} \text {. }
$$

The desired solution to the Gelfand problem then follows from a simple resolvent lemma (see $[\mathbf{3 8}$, Lem 2.1.10]) which shows that

$$
\text { Lat } V=\operatorname{Lat}(I-V)(I+V)^{-1}
$$

and hence Lat $V$ is isomorphic to the linearly ordered lattice for the compressed shift.

The unitary operator $\mathcal{W}: L^{2}[0,1] \longrightarrow H^{2} \ominus \varphi H^{2}$ is therefore useful in passing between properties of the Volterra operator $V$ and the compressed shift $S$. We will use this unitary transformation to investigate the complex symmetric properties of the compressed shift using known properties of the Volterra operator. For instance, a straightforward computation based on the fact that

$$
\left[V^{*} f\right](x)=\int_{x}^{1} f(t) d t
$$

shows that the Volterra operator is complex symmetric: 
Lemma 4.1. The Volterra integration operator $V: L^{2}[0,1] \longrightarrow L^{2}[0,1]$ is $\mathcal{C}$-symmetric with respect to the conjugation operator $[\mathcal{C} f](x)=\overline{f(1-x)}$ on $L^{2}[0,1]$.

As noted in $[\mathbf{2 4}]$, the equation $\mathcal{C V}=V^{*} \mathcal{C}$ ultimately reflects the functional symmetry $K(x, y)=K(1-y, 1-x)$ of the Volterra kernel $K(x, y)$, the characteristic function of the triangle

$$
\{(x, y): 0 \leq y \leq x \leq 1\} .
$$

Equivalently, one might say that the $\mathcal{C}$-symmetry of $V$ arises from the geometric symmetry of the triangle.

Since $V$ is a $\mathcal{C}$-symmetric operator, it follows (by passing to the limit in Lemma 2.13) that the Cayley transform $(I-V)(I+V)^{-1}$ of $V$ is also $\mathcal{C}$-symmetric. Since this operator is unitarily equivalent (via $\mathcal{W}$ ) to the compressed shift $S$, it follows that $S$ is a complex symmetric operator with respect to the conjugation operator $\mathcal{W C W} \mathcal{W}^{*}$ on $H^{2} \ominus \varphi H^{2}$. Remarkably, the corresponding conjugation on $H^{2} \ominus \varphi H^{2}$ is precisely the conjugation $C f=\overline{f z} \varphi$ discussed in the preceding pages.

LEMMA 4.2. The conjugation operator

$$
[\mathcal{C} g](x)=\overline{g(1-x)}
$$

on $L^{2}[0,1]$ and the conjugation operator

$$
C f=\bar{f} z \varphi
$$

on $H^{2} \ominus \varphi H^{2}$ are related by the unitary operator $\mathcal{W}$ :

$$
C=\mathcal{W C} \mathcal{W}^{*} \text {. }
$$

Proof. We prove the lemma by establishing that $\mathcal{W} C=C \mathcal{W}$. For any $g$ in $L^{2}[0,1]$, the integrands of the following integrals are all dominated by $\max \{|g(t)|,|g(1-t)|\}$ and it follows that

$$
\begin{aligned}
{[\mathcal{W C} g](z) } & =\frac{\sqrt{2} i}{z-1} \int_{0}^{1}[\mathcal{C} g](t)[\varphi(z)]^{t} d t \\
& =\frac{\sqrt{2} i}{z-1} \int_{0}^{1} \overline{g(1-t)}[\varphi(z)]^{t} d t \\
& =\frac{\sqrt{2} i}{z-1} \int_{0}^{1} \overline{g(s)}[\varphi(z)]^{1-s} d s \\
& =\bar{z} \varphi(z) \frac{\sqrt{2} i}{1-\bar{z}} \int_{0}^{1} g(s)[\varphi(z)]^{s} d s \\
& =\overline{[\mathcal{W} g](z) z \varphi(z)}
\end{aligned}
$$

for almost every $z$ on $\partial \mathbb{D}$.

Using the unitary transformation $\mathcal{W}: L^{2}[0,1] \longrightarrow H^{2} \ominus \varphi H^{2}$, we can now transform $\mathcal{C}$-real bases in $L^{2}[0,1]$ into $C$-real bases in the model space $H^{2} \ominus \varphi H^{2}$. 
4.2. Constructing $C$-real bases. As we have noted before, one of the primary difficulties in working with model spaces is the lack of convenient orthonormal bases. On the other hand, the space $L^{2}[0,1]$ has many natural orthonormal bases, the most obvious being the exponential basis $\left(e^{2 \pi i n x}\right)_{n \in \mathbb{Z}}$. Since the Sarason transform (4.1) is a unitary operator from $L^{2}[0,1]$ onto $H^{2} \ominus \varphi H^{2}$, it follows that the image of any orthonormal basis in $L^{2}[0,1]$ will be an orthonormal basis for $H^{2} \ominus \varphi H^{2}$. Moreover, Lemma 4.2 guarantees that the image under $\mathcal{W}$ of a $\mathcal{C}$-real basis of $L^{2}[0,1]$ will be $C$-real in $H^{2} \ominus \varphi H^{2}$. In particular, this will provide "natural" $C$-real bases for the model space $H^{2} \ominus \varphi H^{2}$ with respect to which the compressed shift can be represented as a complex symmetric matrix.

We will now construct a continuous family (indexed by a parameter $\alpha$ ) of $C$-real bases for $H^{2} \ominus \varphi H^{2}$ by transforming the $\mathcal{C}$-real bases of $L^{2}[0,1]$ provided by the following lemma:

LEMma 4.3. For each fixed $\alpha$ in $[0,2 \pi)$, the vectors $\left(e_{n}\right)_{n \in \mathbb{Z}}$ defined by

$$
e_{n}(x)=\exp \left[i(\alpha+2 \pi n)\left(x-\frac{1}{2}\right)\right]
$$

form a $C$-real basis of $L^{2}[0,1]$.

Proof. A direct computation shows that each $e_{n}$ is fixed by $C$.

$$
\begin{aligned}
{\left[C e_{n}\right](x) } & =\overline{e^{i(\alpha+2 \pi n)\left((1-x)-\frac{1}{2}\right)}} \\
& =e^{-i(\alpha+2 \pi n)\left(\frac{1}{2}-x\right)} \\
& =e^{i(\alpha+2 \pi n)\left(x-\frac{1}{2}\right)} \\
& =e_{n}(x) .
\end{aligned}
$$

To see that $\left(e_{n}\right)_{n \in \mathbb{Z}}$ is an orthonormal basis for $L^{2}[0,1]$, simply observe the identity

$$
\begin{aligned}
e^{i(\alpha+2 \pi n)\left(x-\frac{1}{2}\right)} & =e^{i\left(\alpha x+2 \pi n x-\frac{\alpha}{2}-\pi n\right)} \\
& =(-1)^{n} e^{-\frac{i}{2} \alpha} e^{i \alpha x} e^{i 2 \pi n x}
\end{aligned}
$$

and note that multiplication by $e^{i \alpha x}$ is a unitary operator on $L^{2}[0,1]$.

For each $\alpha$ in $[0,2 \pi)$, we obtain an orthonormal basis $\left(e_{n}\right)_{n \in \mathbb{Z}}$ of $L^{2}[0,1]$ that is fixed by $\mathcal{C}$. In particular, for each $\alpha$ we obtain a natural basis of $L^{2}[0,1]$ with respect to which $\mathcal{C}$-symmetric operators, such as the Volterra operator and its Cayley transform, have symmetric matrix representations. We now wish to compute the image of this basis in $H^{2} \ominus \varphi H^{2}$ under the unitary transformation $\mathcal{W}$.

Writing each basis vector $e_{n}$ in the form

$$
e_{n}(x)=e^{-i(\alpha+2 \pi n) / 2} e^{i(\alpha+2 \pi n) x},
$$

we see that it suffices to compute $e^{-i \gamma / 2} \mathcal{W} e^{i \gamma t}$ for real $\gamma$. Once this is done, we need only substitute $\gamma=\alpha+2 \pi n$ to find the corresponding basis for our model space. Since this computation is somewhat lengthy, we will present it through a sequence of lemmas. 
The following lemma already shows that for a particular $\alpha$, the image of the basis $\left(e_{n}\right)_{n \in \mathbb{Z}}$ in $H^{2} \ominus \varphi H^{2}$ will be a family of reproducing kernels corresponding to a sequence of points on the unit circle. Since $\varphi$ is analytically continuable across any arc of the unit circle $\partial \mathbb{D}$ not containing $z=1$, the reproducing kernel corresponding to any boundary point $\zeta \neq 1$ in $\partial \mathbb{D}$ is well-defined and belongs to $H^{2} \ominus \varphi H^{2}$.

LEMma 4.4. If $\gamma$ is real, then

$$
\left[\mathcal{W} e^{i \gamma x}\right](z)=\frac{(1-\zeta)}{\sqrt{2} i \zeta} K_{\zeta}(z),
$$

where $K_{\zeta}(z)$ is the reproducing kernel (3.6) for $H^{2} \ominus \varphi H^{2}$ corresponding to the point $\zeta$ on $\partial \mathbb{D}$ defined by

$$
\zeta=\frac{\gamma+i}{\gamma-i}
$$

In particular, we have $\varphi(\zeta)=e^{-i \gamma}$.

Proof. Fixing $\gamma$ in $\mathbb{R}$ and using the definition (4.1) of $\mathcal{W}$ we find that

$$
\begin{aligned}
{\left[\mathcal{W} e^{i \gamma x}\right](z) } & =\frac{\sqrt{2} i}{z-1} \int_{0}^{1} e^{i \gamma t} \exp \left(t \frac{z+1}{z-1}\right) d t \\
& =\frac{\sqrt{2} i}{z-1} \int_{0}^{1} \exp \left[t\left(i \gamma+\frac{z+1}{z-1}\right)\right] d t \\
& =\frac{\sqrt{2} i}{z-1} \cdot \frac{1}{i \gamma+\frac{z+1}{z-1}}\left(\exp \left[\left(i \gamma+\frac{z+1}{z-1}\right)\right]-1\right) \\
& =\frac{\sqrt{2} i}{i \gamma(z-1)+(z+1)}\left(e^{i \gamma} \varphi(z)-1\right) \\
& =\frac{-\sqrt{2} i}{(1-i \gamma)+(1+i \gamma) z}\left(1-\overline{e^{-i \gamma}} \varphi(z)\right) \\
& =\frac{-\sqrt{2} i}{1-i \gamma} \cdot \frac{1}{1+\frac{1+i \gamma}{1-i \gamma} z}\left(1-\overline{e^{-i \gamma}} \varphi(z)\right) .
\end{aligned}
$$

Seeking to construct a scalar multiple of a reproducing kernel from (4.4), we wish to write $e^{-i \gamma}=\varphi(\zeta)$ for some $\zeta$. Defining $\zeta$ by

$$
\gamma=i \frac{\zeta+1}{\zeta-1} \quad \Longleftrightarrow \quad \zeta=\frac{\gamma+i}{\gamma-i},
$$

we see that $\zeta$ lies on the unit circle $\partial \mathbb{D}, \varphi(\zeta)=e^{-i \gamma}$, and that

$$
-\bar{\zeta}=\frac{1+i \gamma}{1-i \gamma} .
$$

Substituting these values into (4.4) shows that

$$
\left[\mathcal{W} e^{i \gamma x}\right](z)=\frac{-\sqrt{2} i}{1-i \gamma} \cdot \frac{1-\overline{\varphi(\zeta)} \varphi(z)}{1-\bar{\zeta} z}
$$




$$
\begin{aligned}
& =\frac{-\sqrt{2} i}{1+\frac{\zeta+1}{\zeta-1}} K_{\zeta}(z) \\
& =\frac{-\sqrt{2} i(\zeta-1)}{(\zeta-1)+(\zeta+1)} K_{\zeta}(z) \\
& =\frac{-\sqrt{2} i(\zeta-1)}{2 \zeta} K_{\zeta}(z) \\
& =\frac{(1-\zeta)}{\sqrt{2} i \zeta} K_{\zeta}(z),
\end{aligned}
$$

where $K_{\zeta}(z)$ denotes the reproducing kernel (3.6) evaluated at the boundary point $\zeta$. This proves the desired formula.

It is important to note that in Clark's paper [10], it is simply noted that $\mathcal{W}$ maps the exponential functions $e^{i \gamma t}$ onto constant multiples of reproducing kernels corresponding to certain points on $\partial \mathbb{D}$. We emphasize here that the exact constants that appear are the key to the complex symmetry. By keeping careful track of the constants that appear in the following computations, we will ultimately be able to follow the symmetry through from $L^{2}[0,1]$ to $H^{2} \ominus \varphi H^{2}$.

Since $\mathcal{W}$ is a unitary transformation, the image of $\mathcal{W} e^{i \gamma x}$ in $H^{2} \ominus \varphi H^{2}$ must be a unit vector. This suggests that we must simplify the expression (4.6) further and examine the constant appearing there more carefully.

LEMMA 4.5. If $\gamma$ is real, then

$$
\left[\mathcal{W} e^{i \gamma x}\right](z)=\frac{(1-\zeta)}{|1-\zeta| \zeta i} k_{\zeta}(z),
$$

where $k_{\zeta}(z)$ is the normalized reproducing kernel for $H^{2} \ominus \varphi H^{2}$ corresponding to $\zeta$.

Proof. Since $\gamma \neq \infty$, we have $\zeta \neq 1$ and the norm of the function $K_{\zeta}$ can be explicitly computed in terms of $\varphi$. In fact, if $z$ approaches $\zeta$ nontangentially we have

$$
\begin{aligned}
\left\|K_{\zeta}\right\|^{2} & =\lim _{z \longrightarrow \zeta}\left\langle K_{z}, K_{z}\right\rangle \\
& =\lim _{z \longrightarrow \zeta} \frac{1-|\varphi(z)|^{2}}{1-|z|^{2}} \\
& =\lim _{z \longrightarrow \zeta} \frac{1-|\varphi(z)|}{1-|z|} \cdot \frac{1+|\varphi(z)|}{1+|z|} \\
& =\lim _{z \longrightarrow \zeta} \frac{1-|\varphi(z)|}{1-|z|} \\
& =\left|\varphi^{\prime}(\zeta)\right| .
\end{aligned}
$$


The interested reader may consult $[\mathbf{3 9}]$ for further details. A straightforward computation using the definition of $\varphi$ shows that

$$
\left\|K_{\zeta}\right\|=\frac{\sqrt{2}}{|1-\zeta|} .
$$

With this computation in mind, we continue from (4.6) and find that

$$
\begin{aligned}
{\left[\mathcal{W} e^{i \gamma x}\right](z) } & =\frac{(1-\zeta)}{|1-\zeta| \zeta i} \frac{|1-\zeta|}{\sqrt{2}} K_{\zeta}(z) \\
& =\frac{(1-\zeta)}{|1-\zeta| \zeta i} k_{\zeta}(z)
\end{aligned}
$$

where $k_{\zeta}$ denotes the normalized kernel function corresponding to $\zeta$. This proves the desired formula.

Although it is evident from the preceding lemma that $\mathcal{W} e^{i \gamma x}$ is a unit vector in $H^{2} \ominus \varphi H^{2}$, this was not our primary objective. Indeed, recall that we wanted to show that for any real $\gamma$, the function $e^{-i \gamma / 2} \mathcal{W} e^{i \gamma x}$ is fixed by $C$. It turns out that the apparently unwieldy constant

$$
\frac{(1-\zeta)}{|1-\zeta| \zeta i}
$$

in preceding formulas appears there for exactly this reason.

The square of (4.7) equals

$$
\frac{-(1-\zeta)^{2}}{(1-\zeta)(1-\bar{\zeta}) \zeta^{2}}=\bar{\zeta}
$$

and hence the constant (4.7) is simply one of the square roots of $\bar{\zeta}$. The particular branch of the square root of $\bar{\zeta}$ represented by (4.7) is not particularly important for our purposes (although it is easily computable for a given $\gamma$ ) and we henceforth denote the constant $(4.7)$ by $\bar{\zeta}^{1 / 2}$.

What we have shown so far is that for any real $\gamma$,

$$
\left[\mathcal{W} e^{i \gamma x}\right](z)=\bar{\zeta}^{1 / 2} k_{\zeta}(z)
$$

where the unimodular constant $\zeta$ satisfies $\varphi(\zeta)=e^{-i \gamma}$. To complete the evaluation of $e^{-i \gamma / 2} \mathcal{W} e^{i \gamma t}$, we simply use (4.5) to describe the value of the constant $e^{-i \gamma / 2}$ in terms of the inner function $\varphi$ :

$$
e^{-i \gamma / 2}=e^{\frac{1}{2} \frac{\zeta+1}{\zeta-1}}=[\varphi(\zeta)]^{\frac{1}{2}} .
$$

As before, we are not concerned with the particular branch of the square root that appears in the preceding formula. We summarize our computations in the following lemma:

LEMMA 4.6. If $\gamma$ is real, then

$$
\left.e^{-i \gamma / 2}\left[\mathcal{W} e^{i \gamma x}\right](z)=\bar{\zeta} \varphi(\zeta)\right]^{\frac{1}{2}} k_{\zeta}(z)
$$


where $k_{\zeta}(z)$ is the normalized reproducing kernel for $H^{2} \ominus \varphi H^{2}$ corresponding to the point $\zeta$. Each function (4.8) is fixed by the conjugation operator $C f=\overline{f z \varphi}$ on $H^{2} \ominus \varphi H^{2}$.

Proof. The first portion of the lemma is simply a summary of the preceding computations. Only the last statement requires proof. If $|z|=1$ and $z \neq 1$, then

$$
\begin{aligned}
k_{\zeta}(z) \sqrt{k_{\zeta}(z)} & =\frac{1-\overline{\varphi(\zeta)} \varphi(z)}{1-\bar{\zeta} z} \cdot \frac{1-\zeta \bar{z}}{1-\varphi(\zeta) \overline{\varphi(z)}} \\
& =\frac{1-\overline{\varphi(\zeta)} \varphi(z)}{1-\varphi(\zeta) \overline{\varphi(z)}} \cdot \frac{1-\zeta \bar{z}}{1-\overline{\zeta z}} \\
& =\overline{\varphi(\zeta) \varphi(z)}\left(\frac{\varphi(\zeta) \overline{\varphi(z)}-1}{1-\varphi(\zeta) \overline{\varphi(z)}}\right) \cdot \zeta \bar{z}\left(\frac{\bar{\zeta} z-1}{1-\bar{\zeta} z}\right) \\
& =\overline{\zeta(\zeta \bar{z} \varphi(z)}
\end{aligned}
$$

since $\varphi(z)$ is unimodular. This identity, along with a short calculation, shows that the function $[\bar{\zeta} \varphi(\zeta)]^{\frac{1}{2}} k_{\zeta}(z)$ is fixed by $C$.

4.3. Summary. Putting this all together, for each fixed $\alpha$ in $[0,2 \pi)$ we obtain an orthonormal basis $\left(e_{n}\right)_{n \in \mathbb{Z}}$ of $L^{2}[0,1]$ defined by $(4.2)$ :

$$
e_{n}(x)=\exp \left[i(\alpha+2 \pi n)\left(x-\frac{1}{2}\right)\right] \text {, }
$$

which is fixed by the conjugation operator

$$
[\mathcal{C} f](x)=\overline{f(1-x)}
$$

on $L^{2}[0,1]$. In light of (4.8), the image of this basis in $H^{2} \ominus \varphi H^{2}$ under the Sarason transform

$$
[\mathcal{W} g](z)=\frac{\sqrt{2} i}{z-1} \int_{0}^{1} g(t)[\varphi(z)]^{t} d t
$$

is given by

$$
\begin{aligned}
{\left[\mathcal{W} e_{n}\right](z) } & =\left[\overline{\zeta_{n}} \varphi\left(\zeta_{n}\right)\right]^{1 / 2} k_{\zeta_{n}}(z) \\
& ={\overline{\zeta_{n}}}^{1 / 2} e^{-\frac{i}{2} \gamma} k_{\zeta_{n}}(z),
\end{aligned}
$$

where $k_{\zeta_{n}}$ denotes the normalized reproducing kernel corresponding (via (4.5)) to the point

$$
\zeta_{n}=\frac{(\alpha+2 \pi n)+i}{(\alpha+2 \pi n)-i}
$$

on the unit circle. The functions $\mathcal{W} e_{n}$ are fixed by the conjugation operator

$$
C f=\bar{f} z \varphi
$$

on $H^{2} \ominus \varphi H^{2}$ and hence the matrix representation of any compressed Toeplitz operator (including the compressed shift) on $H^{2} \ominus \varphi H^{2}$ with respect to the 
basis $\left(\mathcal{W} e_{n}\right)_{n \in \mathbb{Z}}$ will be complex symmetric. Finally, the points $\zeta_{n}$ can be characterized as the inverse image of $e^{-i \alpha}$ under $\varphi$ :

$$
\varphi\left(\zeta_{n}\right)=e^{-i \alpha}
$$

for all $n$ in $\mathbb{Z}$.

In short, we have constructed a continuous (indexed by the parameter $\alpha$ ) family of $C$-real bases for the model space $H^{2} \ominus \varphi H^{2}$. Each such basis naturally corresponds to a "level set" $\left(\zeta_{n}\right)_{n \in \mathbb{Z}}$ of the original inner function. The members of our bases are unimodular scalar multiples of the corresponding normalized reproducing kernels $k_{\zeta_{n}}$.

Our computations in this special case illustrate a more general phenomenon, namely that one can often obtain a continuous family of $C$-real bases for a model space by looking at appropriate level sets of the associated inner function. The key to the present construction was the integral transform $\mathcal{W}$, which mapped $L^{2}[0,1]$ unitarily onto the model space $H^{2} \ominus \varphi H^{2}$. Although generalizations of this integral transform exist for general inner functions (see $[3]$ ), the details of our construction quickly become too cumbersome. Indeed, the lengthy algebraic manipulations in the case of our simple atomic inner function should be enough to convince the reader that a different approach is necessary.

To develop a more general procedure to construct $C$-real bases for model spaces, we will need to appeal to several structure theorems for complex symmetric operators.

\section{Compressed Toeplitz Operators}

In this section, we recall some of the elementary properties of Toeplitz operators and their compressions to model spaces. The most important result of this section is Theorem 5.1 , which shows that compressed Toeplitz operators are complex symmetric.

5.1. Toeplitz operators. For each $u$ in $L^{\infty}(\partial \mathbb{D})$, the Toeplitz operator with symbol $u$ is the operator $T_{u}: H^{2} \rightarrow H^{2}$ defined by $T_{u} f=P(u f)$ where $P$ denotes the orthogonal projection from $L^{2}$ onto $H^{2}$. Note that if $u$ belongs to $H^{\infty}$, then $T_{u}$ is simply the operator $M_{u}$ of multiplication by $u$. It is not hard to show that the adjoint of a Toeplitz operator is given by the simple formula $T_{u}^{*}=T_{\bar{u}}$.

The most important examples of Toeplitz operators arise from the function $u(z)=z$. In this case, $T_{z}$ and $T_{z}^{*}$ are simply the unilateral shift operator $M_{z}$ and the backward shift operator $M_{z}^{*}$, respectively. Indeed, a straightforward computation shows that $T_{z} f=z f$ shifts the Taylor coefficients (at the origin) of $f$ to the right, while

$$
\left[T_{z}^{*} f\right](z)=\frac{f(z)-f(0)}{z}
$$

shifts them to the left. 
A compressed Toeplitz operator is an operator of the form $P_{\varphi} T_{u} P_{\varphi}$ where $T_{u}$ is a standard Toeplitz operator and $P_{\varphi}$ denotes the orthogonal projection from $H^{2}$ onto a model space $H^{2} \ominus \varphi H^{2}$. With a slight abuse of notation, we regard compressed Toeplitz operators as operators acting on the space $H^{2} \ominus \varphi H^{2}$, rather than on $H^{2}$ itself.

5.2. The Backward Shift. Recall (from Example 2.14) that the backward shift $M_{z}^{*}$ is not a complex symmetric operator. That is, there does not exist a conjugation $C$ with respect to which $M_{z}^{*}$ is $C$-symmetric. Indeed, this operator is about as far from complex symmetric as possible, for it violates almost any necessary criterion for being complex symmetric that one can devise.

With respect to the orthonormal basis- $\left(z^{n}\right)_{n=0}^{\infty}$ for $H^{2}$, we can represent $M_{z}^{*}$ as an infinite matrix:

$$
\left(\begin{array}{ccc|ccc}
0 & 1 & 0 & 0 & 0 & \cdots \\
0 & 0 & 1 & 0 & 0 & \cdots \\
0 & 0 & 0 & 1 & 0 & \cdots \\
\hline 0 & 0 & 0 & 0 & 1 & \cdots \\
0 & 0 & 0 & 0 & 0 & \cdots \\
\vdots & \vdots & \vdots & \vdots & \vdots &
\end{array}\right)\left(\begin{array}{c}
a_{0} \\
a_{1} \\
a_{2} \\
a_{3} \\
a_{4} \\
\vdots
\end{array}\right)=\left(\begin{array}{c}
a_{1} \\
a_{2} \\
a_{3} \\
a_{4} \\
a_{5} \\
\vdots
\end{array}\right) .
$$

For the sake of illustration, we have isolated the upper-left $3 \times 3$ block of the matrix above. We immediately recognize this corner as the Jordan block (with $\lambda=0$ )

$$
\left(\begin{array}{lll}
0 & 1 & 0 \\
0 & 0 & 1 \\
0 & 0 & 0
\end{array}\right)
$$

which is a complex symmetric operator (see Example 2.2). Indeed, this is a finite Toeplitz matrix and we have already noted that all finite Toeplitz matrices are complex symmetric operators.

In terms of function theory, this Jordan block represents the compression of $M_{z}^{*}$ to the $M_{z}^{*}$-invariant subspace $H^{2} \ominus z^{3} H^{2}$. The symmetry of this Toeplitz matrix with respect to the second diagonal corresponds to the map

$$
C\left(a_{0}+a_{1} z+a_{2} z^{2}\right)=\overline{a_{2}}+\overline{a_{1}} z+\overline{a_{0}} z^{2}
$$

on $H^{2} \ominus z^{3} H^{2}$. In terms of boundary functions, this is simply

$$
C f=\bar{f} z^{2}=\overline{f z} \varphi
$$

where $\varphi=z^{3}$ is our original inner function.

Similar computations, focused on the upper left $n \times n$ block of our infinite Toeplitz matrix, suggest that the compression of the backward shift $M_{z}^{*}$ to the $M_{z}^{*}$-invariant subspaces $H^{2} \ominus \varphi H^{2}$ are complex symmetric operators. The remarkable thing is that this is true in complete generality. 
5.3. C-symmetry of compressed Toeplitz operators. It turns out that compressed Toeplitz operators are complex symmetric operators with respect to the conjugation operator (3.2) on $H^{2} \ominus \varphi H^{2}$. To be explicit, we recall the following theorem from $[\mathbf{2 3}]$ :

THEOREM 5.1. If $\varphi$ is a nonconstant inner function, $u$ belongs to $L^{\infty}(\partial \mathbb{D})$, and $P_{\varphi}$ denotes the orthogonal projection from $H^{2}$ onto $H^{2} \ominus \varphi H^{2}$, then the compression $P_{\varphi} T_{u} P_{\varphi}$ of the Toeplitz operator $T_{u}$ to $H^{2} \ominus \varphi H^{2}$ is $C$ symmetric with respect to the conjugation operator (3.2).

PRoOF. Let $T=P_{\varphi} T_{u} P_{\varphi}$ denote the compressed Toeplitz operator and let $P$ denote the orthogonal projection from $L^{2}$ onto $H^{2}$. If $f$ and $g$ belong to $H^{2} \ominus \varphi H^{2}$, then

$$
\begin{aligned}
\langle C T f, g\rangle & =\langle C g, T f\rangle=\left\langle\Theta_{g}, P_{\varphi} T_{u} P_{\varphi} f\right\rangle \\
& =\left\langle P_{\varphi} C g, T_{u} f\right\rangle=\langle C g, P(u f)\rangle \\
& =\langle P C g, u f\rangle=\langle C g, u f\rangle \\
& =\langle\overline{g z} \varphi, u f\rangle=\langle\overline{f z \varphi}, u g\rangle \\
& =\langle C f, u g\rangle=\left\langle P P_{\varphi} C f, u g\right\rangle \\
& =\left\langle P_{\varphi} C f, T_{u} g\right\rangle=\left\langle C f, P_{\varphi} T_{u} P_{\varphi} g\right\rangle \\
& =\langle C f, T g\rangle=\left\langle T^{*} C f, g\right\rangle .
\end{aligned}
$$

Thus $C T=T^{*} C$ and $T$ is $C$-symmetric.

In light of Theorem 5.1, every compressed Toeplitz operator (including the compression of the unilateral shift) is a complex symmetric operator. In other words:

COROLLARY. If $\varphi$ is a nonconstant inner function, then the model operator $S f=P_{\varphi}(z f)$ is $C$-symmetric with respect to the conjugation operator $(3.2)$.

Moreover, we have:

COROLlaRY. If $\varphi$ is a nonconstant inner function, then there exists an orthonormal basis of $H^{2} \ominus \varphi H^{2}$ with respect to which every compressed Toeplitz operator $P_{\varphi} T_{u} P_{\varphi}$ has a symmetric matrix representation.

PROOF. The corollary follows from Theorem 5.1 along with Lemmas 2.6 and 2.7 .

This again suggests the problem of finding and computing such bases. The remainder of this note concerns the solution to this problem. In the next section we examine a nontrivial example in detail.

\section{Polar Decomposition of $C$-symmetric Operators}

In order to generalize our construction of $C$-real bases for the model spaces $H^{2} \ominus \varphi H^{2}$ (where $\varphi$ denotes a nonconstant inner function), we first require some background material on the polar decomposition of $C$-symmetric 
operators. It turns out that the polar decompositions of complex symmetric operators have many special properties.

Our interest in this subject stems from the fact that compressed shifts are complex symmetric operators (Theorem 5.1). Much of the material in section appears in [24].

6.1. A theorem of Godič and Lucenko. The first result we require is not a statement about polar decompositions, but rather a statement about the structure of unitary operators themselves. The following old theorem of Godic and Lucenko [29] generalizes the simple geometric notion that a planar rotation can be expressed as the product of two reflections:

THEOREM 6.1 (Godič-Lucenko). If $U$ is a unitary operator on a Hilbert space $\mathcal{H}$, then there exist conjugation operators $C$ and $J$ on $\mathcal{H}$ such that $U=C J$.

This theorem is remarkable for, among other things, stating that all unitary operators (on a fixed Hilbert space $\mathcal{H}$ ) can be constructed using essentially a single antilinear operator. Indeed, we know from Lemma 2.6 that any conjugation on $\mathcal{H}$ can be represented as complex conjugation with respect to a certain orthonormal basis. In this sense, $C$ and $J$ are structurally identical objects and the fine structure of the unitary $U$ arises entirely in how two copies of the same object are put together. Of course, one can also say that since $C$ and $J$ are unitarily equivalent objects, the theorem of Godič and Lucenko is quite natural.

The following easy example of such a factorization is somewhat instructive:

Example 6.2. Let $U: \mathbb{C}^{n} \longrightarrow \mathbb{C}^{n}$ be a unitary operator. Suppose that $U$ has $n$ (necessarily unimodular) eigenvalues $\lambda_{1}, \lambda_{2}, \ldots, \lambda_{n}$ with corresponding orthonormal eigenvectors $e_{1}, e_{2}, \ldots, e_{n}$. One may define conjugation operators $C$ and $J$ on $\mathbb{C}^{n}$ by setting

$$
C e_{k}=\lambda_{k} e_{k}, \quad J e_{k}=e_{k}
$$

for $k=1,2, \ldots, n$ and extending this definition antilinearly to all of $\mathbb{C}^{n}$. It is clear from this construction that $U=C J$. By introducing offsetting unimodular parameters in the definitions of $C$ and $J$, one can see that the decomposition of $U$ as the product of conjugation operators is not uniquely determined. For instance, if we desired more symmetry we could select a branch of the square root and use

$$
C e_{k}=\lambda_{k}^{1 / 2} e_{k}, \quad J e_{k}=\bar{\lambda}_{k}^{1 / 2} e_{k}
$$

instead.

This simple finite dimensional example suggests the method of proof for Theorem 6.1. The conjugation $J$ was chosen to commute with the spectral projections of $U$ and hence one suspects that the spectral theorem is ultimately involved. Indeed, by the spectral theorem it suffices to prove 
Theorem 6.1 in the special case where $U$ is the operator of multiplication by $e^{i \theta}$ on a Lebesgue space $L^{2}(\partial \mathbb{D}, \mu)$ where $\mu$ is a finitely supported Borel measure on $\partial \mathbb{D}$.

EXAMPLE 6.3. If $U$ denotes the unitary operator $[U f]\left(e^{i \theta}\right)=e^{i \theta} f\left(e^{i \theta}\right)$ on $L^{2}(\partial \mathbb{D}, \mu)$, then $U=C J$ where

$$
[C f]\left(e^{i \theta}\right)=e^{\frac{i}{2} \theta} \overline{f\left(e^{i \theta}\right)}, \quad[J f]\left(e^{i \theta}\right)=e^{-\frac{i}{2} \theta} \overline{f\left(e^{i \theta}\right)}
$$

for all $f$ in $L^{2}(\partial \mathbb{D}, \mu)$.

The more determined reader may wish to consider the Godič-Lucenko decomposition of the Fourier-Plancherel transform on $L^{2}(-\infty, \infty)$ as well as those of other well-known unitary operators (see [24] and the original paper $[29])$.

The converse of Theorem 6.1 is also true:

Lemma 6.4. If $C$ and $J$ are conjugation operators on a Hilbert space $\mathcal{H}$, then $U=C J$ is a unitary operator. Moreover, $U$ is both $C$-symmetric and $J$-symmetric.

Proof. If $U=C J$, then (by the isometric property of $C$ and $J$ ) it follows that

$$
\left\langle f, U^{*} g\right\rangle=\langle U f, g\rangle=\langle C J f, g\rangle=\langle C g, J f\rangle=\langle f, J C g\rangle
$$

for all $f, g$ in $\mathcal{H}$. Thus $U^{*}=J C$ from which $C U=U^{*} C$ and $J U=U^{*} J$ both follow.

Although we will frequently refer to Godič-Lucenko theorem, we do not actually require it. In fact, we will explicitly construct the Godič-Lucenko decomposition for Clark operators and then use the decomposition to obtain information about their spectral decompositions.

Before proceeding to discuss the structure of Clark operators, we first need to investigate the complex symmetric structure of compressed shifts. To do this, we will need a generalization of Theorem 6.1 to the class of all $C$-symmetric operators.

6.2. The partial isometry is $C$-symmetric. Recall that the polar decomposition $T=U|T|$ of an operator $T$ expresses $T$ uniquely as the product of a positive operator $|T|=\sqrt{T^{*} T}$ (the modulus) and a partial isometry $U$ (the argument) which satisfies $\operatorname{ker} U=\operatorname{ker} T$ and maps the initial space $(\operatorname{ker} T)^{\perp}$ onto the final space $\operatorname{cl}(\operatorname{ran} T)$ (the closure of the range of $T$ ). Moreover, recall that the modulus satisfies $\operatorname{ker}|T|=\operatorname{ker} T$ and $\operatorname{cl}(\operatorname{ran}|T|)=$ $\operatorname{cl}\left(\operatorname{ran} T^{*}\right)$.

The following theorem states that the partial isometry appearing in the polar decomposition of a $C$-symmetric operator is also $C$-symmetric (with respect to the same $C$ ):

THEOREM 6.5. If $T=U|T|$ is the polar decomposition of a $C$-symmetric operator $T$, then the partial isometry $U$ is also $C$-symmetric: $C U=U^{*} C$. 
The proof of the general assertion can be found in $[\mathbf{2 4}]$ and the reader will have no difficulty proving it in the case that $T$ is invertible. Indeed, if $T$ is invertible then we have the obvious explicit formula $U=T\left(T^{*} T\right)^{-\frac{1}{2}}$ for the partial isometry. A standard argument shows that $U$ is the limit of polynomials of the form $T p\left(T^{*} T\right)$ where the polynomial $p(x)$ has real coefficients. It is not hard to see (by first considering terms of the form $\left.T\left(T^{*} T\right)^{n}\right)$ that any operator of the form $T p\left(T^{*} T\right)$ is also $C$-symmetric.

The preceding theorem places severe restrictions on the class of partial isometries that can occur in the polar decomposition of a $C$-symmetric operator. For instance, Example 2.14 shows that the unilateral shift cannot appear in this context.

6.3. Refined polar decomposition. Theorem 6.5 is the key observation that allows us to relate the partial isometry $U$ in the polar decomposition of a $C$-symmetric operator $T$ to the modulus $|T|$ and the conjugation $C$ itself.

We first consider the special case of invertible $C$-symmetric operators before considering the general situation.

Lemma 6.6. If $T$ is an invertible $C$-symmetric operator with polar decomposition $T=U|T|$, then the antilinear operator $J=C U$ is a conjugation operator which commutes with the spectral projections of $|T|$.

Proof. Since $T$ is invertible, the partial isometry $U$ is unitary. By Theorem 6.5, $U$ is $C$-symmetric and it follows that

$$
J=C U=U^{*} C
$$

which shows that $U^{*}=J C$. Since $U$ is unitary, it follows that

$$
J^{2}=(J C)(C J)=U^{*} U=I
$$

and

$$
\langle J f, J g\rangle=\langle C U f, C U g\rangle=\langle U g, U f\rangle=\langle g, f\rangle
$$

for all $f, g$ in $\mathcal{H}$ and thus $J$ is a conjugation operator. Moreover, the equation $C T=T^{*} C$ is equivalent to

$$
C U|T|=|T| U^{*} C,
$$

which is equivalent to $J|T|=|T| J$. This implies that $J$ also commutes with $p(|T|)$ for any polynomial $p(x)$ with real coefficients and hence with every spectral projection of $|T|$.

For invertible $C$-symmetric operators, the factorization described above leads to a substantial refinement of the polar decomposition. Specifically, we may write $T=C J|T|$ where $J$ is a conjugation operator which commutes with $|T|$. This can be viewed as a generalization of Theorem 6.1 since the unitary operator $U$ in the polar decomposition $T=U|T|$ is the product of two conjugation operators. 
A similar construction holds for general $C$-symmetric operators, although some minor difficulties arise. The fundamental problem is that the partial isometry $U$ in the polar decomposition $T=U|T|$ of an arbitrary $C$-symmetric operator need not be unitary and it becomes necessary to consider so-called partial conjugations. The details, which essentially reduce to glorified bookkeeping of initial spaces and final spaces, can be found in [24]. Fortunately, these issues do not present a major obstacle and one can obtain the following broad generalization of the theorem (Theorem 6.1) of Godic and Lucenko:

THEOREM 6.7. If $T$ is a $C$-symmetric operator, then $T=C J|T|$ where $J$ is a conjugation operator which commutes with the spectral projections of $|T|$.

By insisting that $J$ is supported on all of $\mathcal{H}$ (rather than $\mathrm{cl}(\operatorname{ran}|T|)$, where the partial isometry $U$ is supported), one in general can no longer assert that $U$ is given by the formula $U=C J$. Indeed, if $C$ and $J$ were conjugation operators, then $U$ would be unitary, which is not always the case. For our purposes, however, these sacrifices are not significant and Theorem 6.7 will be sufficient.

\section{Polar decomposition of compressed shifts}

In this section we compute the polar decomposition of the compression of the unilateral shift to the subspace $H^{2} \ominus \varphi H^{2}$ corresponding to a nonconstant inner function $\varphi$. Furthermore, we also compute the decomposition guaranteed by Theorem 6.7. In fact, we also are able to consider a slight generalization of the compressed shift with little additional effort. Namely, we will examine the compression of the operator of multiplication by a disk automorphism to $H^{2} \ominus \varphi H^{2}$.

The most interesting aspect of these computations is the involvement of Clark-type unitary operators, for which we obtain an explicit Godič-Lucenko decomposition (see Theorem 6.1). In Section 8, we will study generalized Clark operators and the following computations will be necessary to consider the eigenstructures of these operators.

7.1. Preliminaries. Since the computations in this section and the next will be quite involved, let us collect some of the notational conventions we have adopted earlier in this note.

For each $\lambda$ in the open unit disk $\mathbb{D}$, we let $b_{\lambda}$ denote the disk automorphism vanishing at $\lambda$ :

$$
b_{\lambda}(z)=\frac{z-\lambda}{1-\bar{\lambda} z} .
$$

We also require the reproducing kernels $K_{\lambda}$ and the corresponding conjugate kernels $C K_{\lambda}$ for $H^{2} \ominus \varphi H^{2}$ :

$$
K_{\lambda}(z)=\frac{1-\overline{\varphi(\lambda)} \varphi(z)}{1-\bar{\lambda} z}, \quad\left[C K_{\lambda}\right](z)=\frac{\varphi(z)-\varphi(\lambda)}{z-\lambda} .
$$


Furthermore, we will frequently refer to the normalized kernel functions

$$
k_{\lambda}=K_{\lambda} /\left\|K_{\lambda}\right\|, \quad C k_{\lambda}=C K_{\lambda} /\left\|K_{\lambda}\right\| .
$$

For each $\lambda$ in $\mathbb{D}$, we consider the compression

$$
S_{\lambda} f=P_{\varphi}\left(b_{\lambda} f\right)
$$

of the operator of multiplication by $b_{\lambda}$ to $H^{2} \ominus \varphi H^{2}$. Here, as before, $P_{\varphi}$ denotes the orthogonal projection from $H^{2}$ onto $H^{2} \ominus \varphi H^{2}$. The operators $S_{\lambda}$ are therefore straightforward generalizations of the compressed shift operator

$$
S_{0} f=P_{\varphi}(z f),
$$

otherwise known as the model or Jordan operator.

We can also say that $S_{\lambda}$ is the compression of the analytic Toeplitz operator $T_{b_{\lambda}}$ to the $M_{z}^{*}$-invariant subspace $H^{2} \ominus \varphi H^{2}$. Since $T_{b_{\lambda}}^{*}=T_{\overline{b_{\lambda}}}$, the adjoint of $S_{\lambda}$ is given by

$$
S_{\lambda}^{*} f=P_{\varphi}\left(\overline{b_{\lambda}} f\right) .
$$

As a special case of Theorem 5.1, it follows that the operators $S_{\lambda}$ are all $C$-symmetric with respect to the conjugation operator (3.2) on $H^{2} \ominus \varphi H^{2}$. In other words, we have

$$
C S_{\lambda}=S_{\lambda}^{*} C
$$

for every $\lambda$ in $\mathbb{D}$.

Our aim in this section is to explicitly compute the factors in the refined polar decomposition (guaranteed by Theorem 6.7)

$$
S_{\lambda}=C J_{\lambda}\left|S_{\lambda}\right|
$$

for each of these operators. Throughout our computations, we will require a simple computational lemma, which generalizes [10, Lem. 2.1].

Lemma 7.1. For each $\lambda$ in $\mathbb{D}$, the following statements hold:

(1) $S_{\lambda} f=b_{\lambda} f$ if and only if $f$ is orthogonal to $C k_{\lambda}$.

(2) $S_{\lambda}^{*} f=f / b_{\lambda}$ if and only if $f$ is orthogonal to $k_{\lambda}$.

Proof. Clearly $S_{\lambda}^{*} f=f / b_{\lambda}$ if and only if $f / b_{\lambda}$ belongs to $H^{2}$. This happens if and only if $f(\lambda)=0$, or equivalently, if and only if $\left\langle f, k_{\lambda}\right\rangle=0$. By the preceding, $S_{\lambda}^{*} C f=(C f) / b_{\lambda}$ if and only if $\left\langle C f, k_{\lambda}\right\rangle=0$, or equivalently, if and only if $f$ is orthogonal to $C k_{\lambda}$. Since $C S_{\lambda}=S_{\lambda}^{*} C$, this implies that $S_{\lambda} f=C\left[C f / b_{\lambda}\right]=b_{\lambda} f$ if and only if $f$ is orthogonal to $C k_{\lambda}$.

The preceding lemma stresses the importance of the normalized conjugate kernel $C k_{\lambda}$ in our discussion of the operator $S_{\lambda}$. Lemma 7.1 essentially states that on the orthocomplement of the one-dimensional subspace spanned by $C k_{\lambda}$, the operator $S_{\lambda}$ acts isometrically (since $\left\|b_{\lambda} f\right\|=\|f\|$ for all $f$ ). 
7.2. The modulus $\left|S_{\lambda}\right|$. To find the modulus

$$
\left|S_{\lambda}\right|=\sqrt{S_{\lambda}^{*} S_{\lambda}}
$$

of $S_{\lambda}$, we need only compute the positive operator $S_{\lambda}^{*} S_{\lambda}$. By Lemma 7.1, it follows that if $f$ is orthogonal to $C k_{\lambda}$ (in other words if $C f(\lambda)=0$ ), then

$$
\begin{aligned}
S_{\lambda}^{*} S_{\lambda} f & =S_{\lambda}^{*}\left(b_{\lambda} f\right) \\
& =f .
\end{aligned}
$$

Hence $\left|S_{\lambda}\right|$ restricts to the identity operator on the orthocomplement of the one-dimensional subspace spanned by the function $C k_{\lambda}$. This tells us, among other things, that $\left|S_{\lambda}\right|$ must map the function $C k_{\lambda}$ onto a nonnegative constant multiple of itself. Using the following lemma, we will ultimately be able to compute this constant and hence obtain an explicit formula for $S_{\lambda}$.

LEMma 7.2. $S_{\lambda} C k_{\lambda}=-\varphi(\lambda) k_{\lambda}$.

ProOF. Since multiplication by a positive real constant will not affect the desired formulas, it suffices to prove them with $K_{\lambda}$ in place of $k_{\lambda}$. Using (7.2) and letting $e_{\lambda}=(1-\bar{\lambda} z)^{-1}$ denote the Szegö kernel we find that

$$
\begin{aligned}
S_{\lambda} C K_{\lambda} & =S_{\lambda}\left(\frac{\varphi-\varphi(\lambda)}{z-\lambda}\right) \\
& =P_{\varphi}\left(b_{\lambda} \frac{\varphi-\varphi(\lambda)}{z-\lambda}\right) \\
& =P_{\varphi}\left(\frac{\varphi-\varphi(\lambda)}{1-\bar{\lambda} z}\right) \\
& =P_{\varphi}\left(\varphi e_{\lambda}\right)-\varphi(\lambda) P_{\varphi}\left(e_{\lambda}\right) \\
& =0-\varphi(\lambda)\left\langle e_{\lambda}, K_{z}\right\rangle \\
& =-\varphi(\lambda) \overline{K_{z}(\lambda)} \\
& =-\varphi(\lambda) K_{\lambda} .
\end{aligned}
$$

Hence $S_{\lambda} C k_{\lambda}=-\varphi(\lambda) k_{\lambda}$ as claimed.

For vectors $u, v$ in $H^{2} \ominus \varphi H^{2}$, we will let $u \otimes v$ denote the rank-one operator

$$
(u \otimes v) f=\langle f, v\rangle u .
$$

In particular, note that such an operator is an orthogonal projection if and only if $u=v$ and $\|u\|=1$. Using Lemma 7.2 and the $C$-symmetry of the operator $S_{\lambda}$, we are now in a position to compute its modulus:

THEOREM 7.3. The modulus $\left|S_{\lambda}\right|$ of $S_{\lambda}$ is given by the formula

$$
\left|S_{\lambda}\right|=\left(I-C k_{\lambda} \otimes C k_{\lambda}\right)+|\varphi(\lambda)|\left(C k_{\lambda} \otimes C k_{\lambda}\right) .
$$

The first term represents the orthogonal projection onto the orthocomplement of the one-dimensional subspace spanned by $C k_{\lambda}$. 
PROOF. We already noted (in the discussion prior to Lemma 7.2) that $\left|S_{\lambda}\right|$ restricts to the identity operator on the orthocomplement of the onedimensional subspace spanned by $C k_{\lambda}$ and therefore it suffices to consider the action of $\left|S_{\lambda}\right|$ on $C k_{\lambda}$. Applying Lemma 7.2 twice and using the antilinearity of $C$ we see that

$$
\left(S_{\lambda} C\right) k_{\lambda}=|\varphi(\lambda)| k_{\lambda}
$$

However, since the operator $S_{\lambda}$ is $C$-symmetric, it satisfies the equation $C S_{\lambda}^{*}=S_{\lambda} C$ and hence

$$
\begin{aligned}
C S_{\lambda}^{*} S_{\lambda} C k_{\lambda} & =S_{\lambda} C S_{\lambda} C k_{\lambda} \\
& =\left(S_{\lambda} C\right)^{2} k_{\lambda} \\
& =|\varphi(\lambda)| k_{\lambda} .
\end{aligned}
$$

Applying $C$ again we obtain $S_{\lambda}^{*} S_{\lambda} C k_{\lambda}=|\varphi(\lambda)| C k_{\lambda}$ which implies the desired result.

In light of (7.4) and Lemma 7.1, we will henceforth assume that $\varphi(\lambda) \neq 0$, since otherwise the polar decomposition of $S_{\lambda}$ is already evident. Indeed, if $\varphi(\lambda)=0$, then $\operatorname{ker} S_{\lambda}$ equals the one-dimensional subspace spanned by $C k_{\lambda}$ and $S_{\lambda}$ acts isometrically (multiplication by $b_{\lambda}$ ) on the orthocomplement of this subspace. In this case, one sees from (7.4) that $\left|S_{\lambda}\right|$ is not invertible. Rather, $\left|S_{\lambda}\right|$ reduces to the orthogonal projection onto the orthocomplement of the one-dimensional space spanned by $C k_{\lambda}$. The difficulties incurred by this situation are not significant and involve only keeping track of initial and final spaces. Moreover, the partial isometry in the polar decomposition of $S_{\lambda}$ then decomposes as the product $C J_{\lambda}$ where $J_{\lambda}$ is a so-called partial conjugation (see [24]).

The condition $\varphi(\lambda) \neq 0$ actually makes our job slightly more difficult, but more interesting. By insisting that $\varphi(\lambda) \neq 0$, we ensure that $S_{\lambda}$ is invertible. The partial isometry $U_{\lambda}$ appearing in the polar decomposition $S_{\lambda}=U_{\lambda}\left|S_{\lambda}\right|$ is therefore unitary and hence decomposes as the product $C J_{\lambda}$ of two conjugation operators (by Theorem 6.7).

7.3. The conjugation $J_{\lambda}$. By Theorem 6.7 , we may write

$$
S_{\lambda}=C J_{\lambda}\left|S_{\lambda}\right|
$$

where $J_{\lambda}$ is a conjugation operator on $H^{2} \ominus \varphi H^{2}$ which commutes with the spectral projections of $\left|S_{\lambda}\right|$. Thus $J_{\lambda}$ preserves the spectral subspaces of $\left|S_{\lambda}\right|$, namely the one-dimensional span of $C k_{\lambda}$ and its orthocomplement. We can verify this assertions rather explicitly.

THEOREM 7.4. The conjugation operator $J_{\lambda}$ in the refined polar decomposition $S_{\lambda}=C J_{\lambda}\left|S_{\lambda}\right|$ is given by the explicit formula

$$
J_{\lambda} f= \begin{cases}C\left(b_{\lambda} f\right) & f \perp C k_{\lambda} \\ \bar{\alpha} C k_{\lambda} & f=C k_{\lambda}\end{cases}
$$


where the unimodular constant $\alpha$ is given by $\alpha=-\varphi(\lambda) /|\varphi(\lambda)|$.

Proof. To find $J_{\lambda}$, we simply write

$$
J_{\lambda}\left|S_{\lambda}\right|=C S_{\lambda}
$$

and compute the action of $J_{\lambda}$ on the two spectral subspaces of $\left|S_{\lambda}\right|$. If $f \perp C k_{\lambda}$, then $\left|S_{\lambda}\right| f=f$ by (7.4) and hence

$$
J_{\lambda} f=J_{\lambda}\left|S_{\lambda}\right| f=C S_{\lambda} f=C\left(b_{\lambda} f\right)
$$

by (7.6) and Lemma 7.1 . Since $\varphi(\lambda) \neq 0$ (by our nontriviality assumption) we have

$$
\begin{aligned}
|\varphi(\lambda)| J\left(C k_{\lambda}\right) & =J\left|S_{\lambda}\right|\left(C k_{\lambda}\right) \\
& =C\left(S_{\lambda} C k_{\lambda}\right) \\
& =-\overline{\varphi(\lambda)} C k_{\lambda},
\end{aligned}
$$

the two equalities following from (7.6) and Lemma 7.2, respectively. Putting these calculations together yields the desired explicit formula for $J_{\lambda}$.

7.4. The unitary $U_{\lambda}$. We can now compute the partial isometry

$$
U_{\lambda}=C J_{\lambda}
$$

in the polar decomposition $S_{\lambda}=U_{\lambda}\left|S_{\lambda}\right|$ of $S_{\lambda}$ using (7.5). We note that $U_{\lambda}$ is actually unitary since both $C$ and $J_{\lambda}$ are conjugation operators on all of $H^{2} \ominus \varphi H^{2}$ (recall that we are assuming that $\varphi(\lambda) \neq 0$ so that (7.4) does not reduce to a projection).

Applying $C$ to $(7.5)$ yields the piecewise formula

$$
U_{\lambda} f= \begin{cases}b_{\lambda} f & f \perp C k_{\lambda} \\ \alpha k_{\lambda} & f=C k_{\lambda}\end{cases}
$$

where $\alpha$ once again denotes the unimodular constant $\alpha=-\varphi(\lambda) /|\varphi(\lambda)|$. It turns out that $U_{\lambda}$ is actually a rank-one unitary perturbation of the original operator $S_{\lambda}$, although this is not immediately obvious. The following lemma is the key:

LEMma 7.5. $U_{\lambda}$ is given by the formula

$$
U_{\lambda}=S_{\lambda}\left(I-C k_{\lambda} \otimes C k_{\lambda}\right)+\alpha\left(k_{\lambda} \otimes C k_{\lambda}\right) .
$$

Proof. It suffices to verify the formula in the two cases $f \perp C k_{\lambda}$ and $f=C k_{\lambda}$. If $f \perp C k_{\lambda}$, then the operator defined by the right hand side of (7.7) applied to $f$ clearly yields $S_{\lambda} f=b_{\lambda} f$ (using Lemma 7.1). This agrees with $U_{\lambda}$ for such $f$. On the other hand, the operator defined by the right hand side of (7.7) applied to $C k_{\lambda}$ obviously yields $\alpha k_{\lambda}$, which again agrees with $U_{\lambda}$. This establishes the desired formula for $U_{\lambda}$.

Another formula for $U_{\lambda}$ is provided by the following lemma:

LEMma 7.6. $U_{\lambda}$ is given by the formula

$$
U_{\lambda}=S_{\lambda}+(\alpha+\varphi(\lambda))\left(k_{\lambda} \otimes C k_{\lambda}\right) .
$$


Proof. Using (7.7) and the fact that $S_{\lambda} C k_{\lambda}=-\varphi(\lambda) C k_{\lambda}$ (Lemma 7.2), it follows that

$$
\begin{aligned}
U_{\lambda} & =S_{\lambda}-\left(S_{\lambda} C k_{\lambda} \otimes C k_{\lambda}\right)+\alpha\left(k_{\lambda} \otimes C k_{\lambda}\right. \\
& =S_{\lambda}+(\alpha+\varphi(\lambda))\left(k_{\lambda} \otimes C k_{\lambda}\right)
\end{aligned}
$$

which is the desired formula.

We remark at this point that $U_{\lambda}$ is a rank-one, unitary perturbation of $S_{\lambda}$. Moreover, it is $C$-symmetric. Although Theorem 6.5 and Lemma 6.4 assert that $U_{\lambda}$ is automatically $C$-symmetric, this can be seen directly. Indeed, we already know that $S_{\lambda}$ is $C$-symmetric (by Theorem 5.1) and it is easy to verify that the rank-one perturbing operator $\alpha\left(k_{\lambda} \otimes C k_{\lambda}\right)$ is also $C$-symmetric. In fact, it is not hard to show that a rank one operator $u \otimes v$ is $C$-symmetric if and only if it is a constant multiple of $u \otimes C u$.

We summarize our results in the following theorem:

THEOREM 7.7. Let $\varphi$ denote a nonconstant inner function, let $P_{\varphi}$ denote the orthogonal projection from $H^{2}$ onto $H^{2} \ominus \varphi H^{2}$, and let $\lambda$ be a point in $\mathbb{D}$ such that $\varphi(\lambda) \neq 0$. The polar decomposition of the compressed Toeplitz operator

$$
S_{\lambda} f=P_{\varphi}\left(\frac{z-\lambda}{1-\bar{\lambda} z} f\right)
$$

is given by $S_{\lambda}=U_{\lambda}\left|S_{\lambda}\right|$ where $U_{\lambda}$ is the rank-one, unitary, C-symmetric perturbation of $S_{\lambda}$ given by (7.8) and $\left|S_{\lambda}\right|$ is given by (7.4). Moreover, $U_{\lambda}=C J_{\lambda}$ where the auxiliary conjugation operator $J_{\lambda}$ is given by $(7.5)$.

As we mentioned before, one can treat the case $\varphi(\lambda)=0$ similarly, although $U_{\lambda}$ will not longer be unitary and $J_{\lambda}$ will then be only a partial conjugation [24].

\section{Generalized Clark Operators}

The operator $U_{\lambda}$ defined by (7.8) is not the only rank-one $C$-symmetric unitary perturbation of $S_{\lambda}$. Indeed, for any unimodular constant $\alpha$, the operator

$$
U_{\lambda, \alpha}=S_{\lambda}+(\alpha+\varphi(\lambda))\left(k_{\lambda} \otimes C k_{\lambda}\right)
$$

is $C$-symmetric (since $S_{\lambda}$ and $k_{\lambda} \otimes C k_{\lambda}$ are both $C$-symmetric) and unitary, regardless of whether the inner function $\varphi$ vanishes at $\lambda$. That $U_{\lambda, \alpha}$ is unitary can be seen by writing $U_{\lambda, \alpha}$ in a form analogous to (7.7) and using the lemmas of the preceding section.

We refer to operators of the form (8.1) as generalized Clark operators due to their similarity to the operators considered by Clark in $[10]$. The most significant departure from the approach taken there, however, comes in our frequent use of antilinear operators. For instance:

THEOREM 8.1. Each generalized Clark operator $U_{\lambda, \alpha}$ factors as the product of two conjugation operators:

$$
U_{\lambda, \alpha}=C J_{\lambda, \alpha}
$$


where the conjugation operator $J_{\lambda, \alpha}$ is given by

$$
J_{\lambda, \alpha} f= \begin{cases}C\left(b_{\lambda} f\right) & f \perp C k_{\lambda} \\ \bar{\alpha} C k_{\lambda} & f=C k_{\lambda} .\end{cases}
$$

Proof. The proof is almost identical to the proof of Theorem 7.4.

8.1. Eigenstructure of generalized Clark operators. In the traditional approach to the Godič-Lucenko decomposition (Theorem 6.1), one first applies the spectral theorem to a given unitary operator to obtain a representation of that unitary as the product of two conjugations. In the present situation, things are reversed. Theorem 8.1 already provides us with the decomposition $U_{\lambda, \alpha}=C J_{\lambda, \alpha}$ of any generalized Clark operator. We can actually use this representation to explore the eigenstructure of the operators $U_{\lambda, \alpha}$.

THEOREM 8.2. If a function $f$ in $H^{2}$ is a constant multiple of the function

$$
f_{\xi}(z)=\frac{1-\overline{\left(\frac{\alpha+\varphi(\lambda)}{1+\overline{\varphi(\lambda)})}\right)} \varphi(z)}{1-\overline{\left(\frac{\xi+\lambda}{1+\bar{\lambda} \xi}\right)} z},
$$

then $f$ belongs to $H^{2} \ominus \varphi H^{2}$ and is an eigenvector of the generalized Clark operator $U_{\lambda, \alpha}$ corresponding to the eigenvalue $\xi$. Moreover, all eigenvectors are simple and are of the above form. A necessary (but not sufficient) condition for the function (8.4) to belong to $H^{2}$ is that

$$
\varphi\left(\frac{\xi+\lambda}{1+\bar{\lambda} \xi}\right)=\frac{\alpha+\varphi(\lambda)}{1+\overline{\varphi(\lambda)} \alpha}
$$

holds in the sense of nontangential limiting values.

Proof. A function $f$ is an eigenvector of the generalized Clark operator $U_{\lambda, \alpha}=C J_{\lambda, \alpha}$ corresponding to the (necessarily unimodular) eigenvalue $\xi$ if and only if

$$
J_{\lambda, \alpha} f=\bar{\xi} C f .
$$

In light of the explicit formula (8.3) for $J_{\lambda, \alpha}$, we take the orthogonal decomposition of $f$ with respect to the one-dimensional subspace spanned by $C k_{\lambda}$. After possibly multiplying through by a constant, we may assume that $f$ is of the form

$$
f=g+C K_{\lambda}
$$

where $g \perp C K_{\lambda}$. Substituting this into (8.5) we deduce that

$$
J_{\lambda, \alpha}\left(g+C K_{\lambda}\right)=\bar{\xi}\left(C g+K_{\lambda}\right) .
$$

By (8.3), this can be rewritten

$$
C\left(b_{\lambda} g\right)+\bar{\alpha} C K_{\lambda}=\bar{\xi} C g+\bar{\xi} K_{\lambda} .
$$


Applying $C$ to the equation above gives us

$$
b_{\lambda} g+\alpha K_{\lambda}=\xi g+\xi C K_{\lambda} .
$$

Solving for $g$ we find that

$$
g=\frac{\xi C K_{\lambda}-\alpha K_{\lambda}}{b_{\lambda}-\xi} .
$$

We can now solve for the eigenvector $f$ :

$$
\begin{aligned}
f & =g+C K_{\lambda} \\
& =\frac{\xi C K_{\lambda}-\alpha K_{\lambda}}{b_{\lambda}-\xi}+C K_{\lambda} \\
& =\frac{\xi C K_{\lambda}-\alpha K_{\lambda}+b_{\lambda} C K_{\lambda}-\xi C K_{\lambda}}{b_{\lambda}-\xi} \\
& =\frac{b_{\lambda} C K_{\lambda}-\alpha K_{\lambda}}{b_{\lambda}-\xi} .
\end{aligned}
$$

Using the formulas (7.2) for $K_{\lambda}$ and $C K_{\lambda}$ we find that

$$
\begin{aligned}
f & =\frac{\frac{z-\lambda}{1-\bar{\lambda} z} \cdot \frac{\varphi-\varphi(\lambda)}{z-\lambda}-\alpha \frac{1-\overline{\varphi(\lambda)} \varphi}{1-\bar{\lambda} z}}{\frac{z-\lambda}{1-\bar{\lambda} z}-\xi} \\
& =\frac{\frac{\varphi-\varphi(\lambda)}{1-\bar{\lambda} z}-\alpha \frac{1-\overline{\varphi(\lambda)} \varphi}{1-\bar{\lambda} z}}{\frac{z-\lambda-\xi+\xi \bar{\lambda} z}{1-\bar{\lambda} z}} \\
& =\frac{\varphi-\varphi(\lambda)-\alpha+\alpha \overline{\varphi(\lambda)} \varphi}{z-\lambda-\xi+\xi \bar{\lambda} z} \\
& =\frac{\varphi(1+\overline{\varphi(\lambda)} \alpha)-(\alpha+\varphi(\lambda))}{z(1+\bar{\lambda} \xi)-(\lambda+\xi)} \\
& =\left(\frac{\alpha+\varphi(\lambda)}{\lambda+\xi}\right) \frac{1-\left(\frac{1+\overline{\varphi(\lambda)} \alpha}{\alpha+\varphi(\lambda)}\right) \varphi}{1-\left(\frac{1+\bar{\lambda} \xi}{\lambda+\xi}\right) z}
\end{aligned}
$$

and hence $f$ is a constant multiple of the function $f_{\xi}$ defined by (8.4). Conversely, we see that if $\xi$ is a unimodular constant such that $f_{\xi}$ belongs to $H^{2}$, then $f_{\xi}$ is an eigenvector of $U_{\lambda, \alpha}$ corresponding to the eigenvalue $\xi$. Moreover, the computation above shows that the eigenspaces of $U_{\lambda, \alpha}$ are one-dimensional.

A necessary condition for a function of the form (8.4) to belong to $H^{2}$ is that $\varphi$ have the nontangential limiting value $b_{-\varphi(\lambda)}(\alpha)$ at the point $b_{-\lambda}(\xi)$. In other words, the condition

$$
\varphi\left(\frac{\xi+\lambda}{1+\bar{\lambda} \xi}\right)=\frac{\alpha+\varphi(\lambda)}{1+\overline{\varphi(\lambda)} \alpha}
$$


is necessary for $f_{\xi}$ to be an eigenvector of $U_{\lambda, \alpha}$ corresponding to the eigenvalue $\xi$. In general, this condition is not sufficient and we must examine the angular derivative (either directly or via the local Dirichlet integral [39]) of $\varphi$ at the point $b_{-\lambda}(\xi)$. We do not wish to pursue the function theoretic details here and simply remark that (8.6) completely generalizes the original result of Clark [10, Thm. 3.2].

8.2. $C$-real bases for model spaces. We have seen that many natural operators associated with the model spaces are $C$-symmetric. Indeed, we have noted that all compressed Toeplitz operators (including the compressed shift) and all generalized Clark operators (defined by equation (8.1)) are $C$ symmetric.

In general, the spaces $H^{2} \ominus \bar{\varphi} H^{2}$ do not come equipped with "natural" orthonormal bases and hence we must construct them. Recall that we wish to find $C$-real orthonormal bases (those whose vectors are fixed by $C$ ) since, among other things, the matrix representation of a $C$-symmetric operator with respect to a $C$-real basis is symmetric (Lemma 2.7 ).

From this point of view, our interest in the generalized Clark operators $U_{\lambda, \alpha}$ lies in the fact that they are unitary. Under certain circumstances, the eigenvectors of $U_{\lambda, \alpha}$ might furnish a $C$-real orthonormal basis for $H^{2} \ominus \varphi H^{2}$.

We have already computed the Godič-Lucenko decomposition $U_{\lambda, \alpha}=$ $C J_{\lambda, \alpha}$ (Theorem 8.1) for these operators and used it to compute their eigenvalues and eigenvectors (Theorem 8.2). Since the eigenvectors of any generalized Clark operator are simple and pairwise mutually orthogonal, we need only address the question of whether a given $U_{\lambda, \alpha}$ has a complete set of eigenvectors and whether the associated one-dimensional eigenspaces are fixed by $C$.

The first question is essentially a function theoretic one and can be discussed in terms of the analytic properties of the inner function $\varphi$. Several practical conditions guaranteeing that a standard Clark operator has pure point spectrum can be found in $[\mathbf{1 0}]$. We do not wish to go into further detail here, although we do point out that we are now free to vary the parameter $\lambda$ throughout $\mathbb{D}$ (as well as $\alpha$ on $\partial \mathbb{D}$ ) and we only need a single generalized Clark operator $U_{\lambda, \alpha}$ to have pure point spectrum in order to produce a complete set of eigenvectors.

The following general lemma addresses the second question. Namely, it shows that we may select a unit vector, fixed by $C$, from each of the (necessarily one-dimensional) eigenspaces of $U_{\lambda, \alpha}$ :

LEMMA 8.3. If $T$ is a normal $C$-symmetric operator, then the eigenspaces of $T$ are fixed by $C$.

Proof. Since $T$ is normal, the equation $T f=\lambda f$ implies that $T^{*} f=$ $\bar{\lambda} f$. Applying $C$ to the preceding equation implies that $T(C f)=\lambda(C f)$ and hence the eigenspaces of $T$ are invariant under $C$. 
Thus, if $\lambda$ and $\alpha$ are values (in $\mathbb{D}$ and on $\partial \mathbb{D}$, respectively) such that the generalized Clark operator $U_{\lambda, \alpha}$ has pure point spectrum, then we can construct a $C$-real orthonormal basis of $H^{2} \ominus \varphi H^{2}$. Indeed, let $\left(\zeta_{n}\right)_{n=1}^{\operatorname{dim} \mathcal{H}}$ be an enumeration of the points on $\partial \mathbb{D}$ such that

$$
\varphi\left(\zeta_{n}\right)=\frac{\alpha+\varphi(\lambda)}{1+\overline{\varphi(\lambda)} \alpha}
$$

(as nontangential limiting values) and such that the normalized reproducing kernels $k_{\zeta_{n}}$ belong to $H^{2}$ (and hence $H^{2} \ominus \varphi H^{2}$ ).

In this case, the numbers

$$
\xi_{n}=\frac{\zeta_{n}-\lambda}{1-\bar{\lambda} \zeta_{n}}
$$
will be simple eigenvalues for $U_{\lambda, \alpha}$ and corresponding unit eigenvectors are
given by the formula

$$
e_{n}(z)=\left(\overline{\zeta_{n}} \frac{\alpha+\varphi(\lambda)}{1+\varphi(\lambda) \alpha}\right)^{\frac{1}{2}} k_{\zeta_{n}} .
$$

The particular branch of the square root used does not matter and the fact that $C e_{n}=e_{n}$ for all $n$ follows from a computation similar to that in the proof of Lemma 4.6 .

\section{References}

[1] Agmon, S., Sur un problème de translations, C.R. Acad. Sci. Paris, 229 (1949), no.11,

[2] Ahern, P., Clark, D., On inner functions with $H^{p}$ derivative, Michigan Math. J. 21 (1974), 115-127.

[3] Ahern, P., Clark, D., On functions orthogonal to invariant subspaces, Acta Math. 124 (1970), 191-204.

[4] Aleksandrov, A.B., On A-integrability of boundary values of harmonic functions. Mat. Zametki 30 (1981), 59-72.

[5] Bar-On, I., Ryaboy, V., Fast diagonalization of large and dense complex symmetric matrices, with applications to quantum reaction dynamics, SIAM J. Sci. Comput. 18 (1997), no. 5, 1412-1435.

[6] Brodskii, M.S., On a problem of I.M. Gelfand, Uspehi Matem. Nauk, 12 (1957), no. 2, 129-132.

[7] Carathéodory, C., Fejér, L., Über den Zusammenghang der Extremen von harmonischen Funktionen mit ihren Koeffizienten und über den Picard-Landau'schen Satz, Rend. Circ. Mat. Palermo 31 (1911), 218-239.

[8] Cima, J.A., Ross, W.T., The Backward Shift on the Hardy Space, American Mathematical Society, Providence R.I., 2000.

[9] Cima, J.A., Ross, W.T., Matheson, A., The Cauchy Transform, (preprint), [10] Clark, D., One dimensional perturbations of restricted shifts, J. Anal. Math.
25 (1972), 169-191.

[11] Craven, B.D., Complex symmetric matrices, J. Austral. Math. Soc. 10 (1969), 341-

[12] Donoghue,W.F., The lattice of invariant subspaces of a completely continuous quasinilpotent transformation, Pac. J. Math. 7 (1957), 1031-1035.

[13] Douglas, R.G., Shapiro, H.S., Shields, A.L., Cyclic vectors and invariant subspaces for the backward shift operator, Ann. Inst. Fourier (Grenoble) 20 (1970), no.1, 37-76. 
[14] Duren, P.L., Theory of $H^{p}$ Spaces, Pure and Appl. Math., Vol 38, Academic Press, New York, 1970.

[15] Duren, P.L.. Univalent Functions, Springer-Verlag, New York, 1983.

[16] FitzGerald, C.H., Horn, R.A., On the structure of hermitian-symmetric inequalities, J. London Math. Soc, (2) 15 (1977), no. 3, 419-430.

[17] FitzGerald, C.H., Horn, R.A., On quadratic and bilinear forms in function theory, Proc. London Math. Soc. (3) 44 (1982), no. 3, 554-576.

[18] Gantmacher, F.R., The Theory of Matrices (Vol. 2), Chelsea, New York, 1989.

[19] Garcia, S.R., Conjugation, the backward shift, and Toeplitz kernels, (To appear: J. Operator Theory).

[20] Garcia, S.R., Inner matrices and Darlington synthesis, Methods. Funct. Anal. Topology. 11, no, 1, (2005), 37-47.

[21] Garcia, S.R., A *-closed subalgebra of the Smirnov class, Proc. Amer. Math. Soc. 133, no. 7, (2005), 2051-2059.

[22] Garcia, S.R., Prodan, E., Putinar, M., Norm estimates of complex symmetric operators applied to quantum systems, (submitted)

[23] Garcia, S.R., Putinar, M., Complex symmetric operators and applications, (To appear: Trans. Amer. Math. Soc.).

[24] Garcia, S.R., Putinar, M., Complex symmetric operators and applications II, (submitted).

[25] Garcia, S.R., Sarason, D., Real outer functions, Indiana Univ. Math. J., 52 (2003), $1397-1412$.

[26] Gelfand, I.M., A problem (Russian), Uspehi Matem. Nauk, 5 (1938), 233.

[27] Glazman, I.M., Direct methods of the qualitative spectral theory of singular differential operators (Russian), Gos. Iz. Fiz.-Mat. Lit., Moscow, 1963.

[28] Glazman, I.M., An analogue of the extension theory of hermitian operators and a nonsymmetric one-dimensional boundary-value problem on a half-axis (Russian), Dokl. Akad. Nauk SSSR 115 (1957), 214-216.

[29] Godič, V.I., Lucenko, I.E., On the representation of a unitary operator as a product of two involutions (Russian), Uspehi Mat. Nauk 20 (1965), 64-65.

[30] Halmos, P.R., A Hilbert Space Problem Book (Second Edition), Springer-Verlag, 1982.

[31] Helson, H., Large analytic functions, II, Analysis and Partial Differential Equations, Lecture Notes in Pure and Appl. Math., 122, Dekker, New York, 1990, 217-220.

[32] Horn, R.A., Johnson, C.R., Matrix Analysis, Cambridge Univ. Press, Cambridge, 1985.

[33] Hua, L.-K., On the theory of automorphic functions of a matrix variable I: Geometrical basis, Amer. J. Math. 66 (1944), 470-488.

[34] Kalisch, G.K., A functional anaysis proof of Titchmarch's theorem on convolution, J. Math. Anal. Appl., 5 (1962), no.2, 176-183.

[35] Koosis, P., Introduction to $H_{p}$ Spaces. Cambridge University Press, Cambridge, 1998.

[36] Jacobson, N., Normal semi-linear transformations, Amer. J. Math. 61 (1939), 45-58

[37] Nikolski, N.K., Treatise on the Shift Operator, Springer-Verlag, New York, 1986.

[38] Nikolski, N.K., Operators, Functions, and Systems: An Easy Reading, Volume 2: Model Operators and Systems, American Mathematical Society, Providence R.I. 2002.

[39] Richter, S., Sundberg, C., A formula for the local Dirichlet integral, Michigan Math. J. 38 (1991), 355-379.

[40] Ross, W.T., Shapiro, H.S., Generalized Analytic Continuation, University Lecture Series, Volume 25, American Mathematical Society, Providence R.I., 2002.

[41] Sakhnovich, L.A., Spectral analysis of Volterra operators and inverse problems (Russian), Doklady Akad. Nauk SSSR, 115 (1957), no.4, 666-669.

[42] Sarason, D., A remark on the Volterra operator, J. Math. Anal. Appl., 12 (1965) 244-246. 

[43] Schur, I., Ein Satz über Quadratische Formen mit Komplexen Koeffizienten, Amer.
J. Math. 67 (1945), 472-480.

[44] Scott, N.H., A new canonical form for complex symmetric matrices, Proc. Roy. Soc. London Ser. A 441 (1993), no. 1913, 625-640.

[45] Scott, N.H., A theorem on isotropic null vectors and its application to thermoelasticity. Proc. Roy. Soc. London Ser. A 440 (1993), no. 1909, 431-442.

[46] Siegel, C.L., Symplectic geometry, Amer. J. Math. 65 (1943), 1-86.

[47] Takagi, T., On an algebraic problem related to an analytic theorem of Caratheodory and Fejer and on an allied theorem of Landau, Japan J. Math. 1 (1925), 83-93. [48] Walsh, J.L., Interpolation and approximations by rational functions in the complex
domain, Amer. Math. Soc. Coll. Publ. 20., 1935 . [49] Wellstein, J., Über symmetrische, alternierende und orthogonale Normalformen von
Matrizen, J. Reine Angew. Math. 163 (1930), 166-182.

Department of Mathematics, University of CALifornia at SAnta Barbara.

SANTA BARBara, CALIFORNia, 93106-3080

E-mail address: garcias@math.ucsb.edu

URL: http://math.ucsb. edu/ garcias

998.

$-58$.

e:

R.I.,

ath.

ture

Rus-

65), 